\title{
pDCs in lung and skin fibrosis in a bleomycin-induced model and patients with systemic sclerosis
}

\author{
Suzanne Kafaja, ${ }^{1,2}$ Isela Valera, ${ }^{1,2}$ Anagha A. Divekar, ${ }^{1}$ Rajan Saggar, ${ }^{3}$ Fereidoun Abtin, ${ }^{4}$ \\ Daniel E. Furst, ${ }^{2}$ Dinesh Khanna, ${ }^{2}$ and Ram Raj Singh ${ }^{1,2,5,6,7}$
}

${ }^{1}$ Autoimmunity and Tolerance Laboratory, ${ }^{2}$ Division of Rheumatology, ${ }^{3}$ Division of Pulmonary and Critical Care Medicine, Department of Medicine, ${ }^{4}$ Department of Radiological Sciences, ${ }^{5}$ Molecular Toxicology Interdepartmental Program, ${ }^{6}$ Jonsson Comprehensive Cancer Center, and 'Department of Pathology and Laboratory Medicine, David Geffen School of Medicine at UCLA, Los Angeles, California, USA.

Fibrosis is the end result of most inflammatory conditions, but its pathogenesis remains unclear. We demonstrate that, in animals and humans with systemic fibrosis, plasmacytoid DCs (pDCs) are unaffected or are reduced systemically (spleen/peripheral blood), but they increase in the affected organs (lungs/skin/bronchoalveolar lavage). A pivotal role of pDCs was shown by depleting them in vivo, which ameliorated skin and/or lung fibrosis, reduced immune cell infiltration in the affected organs but not in spleen, and reduced the expression of genes and proteins implicated in chemotaxis, inflammation, and fibrosis in the affected organs of animals with bleomycin-induced fibrosis. As with animal findings, the frequency of pDCs in the lungs of patients with systemic sclerosis correlated with the severity of lung disease and with the frequency of $\mathrm{CD}^{+}$and IL-4+ ${ }^{+} \mathrm{T}$ cells in the lung. Finally, treatment with imatinib that has been reported to reduce and/or prevent deterioration of skin and lung fibrosis profoundly reduced pDCs in lungs but not in peripheral blood of patients with systemic sclerosis. These observations suggest a role for $\mathrm{pDCs}$ in the pathogenesis of systemic fibrosis and identify the increased trafficking of pDCs to the affected organs as a potential therapeutic target in fibrotic diseases.

Authorship note: SK, IV, and AAD contributed equally to the work.

Conflict of interest: The authors have declared that no conflict of interest exists.

Submitted: October 30, 2017

Accepted: April 4, 2018

Published: May 3, 2018

\section{Reference information:}

JCI Insight. 2018;3(9):e98380. https://doi.org/10.1172/jici. insight.98380.

\section{Introduction}

Uncontrolled tissue repair or fibrosis is a common end result of tissue injury in a wide range of diseases (1, 2). Fibrotic disorders are estimated to contribute to about $45 \%$ of all-cause mortality in the US (3). Inflammation and fibrosis in multiple organs including skin and lung are hallmarks of systemic sclerosis (SSc) that has no effective treatment. Mechanisms that drive fibrosis remain mostly unclear. Studies in patients and animal models suggest a role for T cells, particularly those that produce type- 2 cytokines such as IL- 4 in the development of fibrosis $(4,5)$. DCs that play a role in T cell priming and differentiation accumulate in the lungs of patients with idiopathic pulmonary fibrosis (6) and in the skin of patients with SSc (7). DCs from SSc patients secrete more cytokines than healthy persons upon TLR stimulation (8). DCs also infiltrate the lungs of animals injected with bleomycin that causes skin and lung fibrosis (9). However, the exact role of DCs in the pathogenesis of fibrosis is unclear.

DCs comprise of heterogeneous population of antigen-presenting cells, including myeloid DCs (mDC), tissue-resident DCs, and plasmacytoid DCs ( $\mathrm{pDC}$ ). These DC subsets may play different roles during different stages of fibrosis development. For example, depletion of non-pDCs that express transcription factor ZBTB46 worsens bleomycin-induced profibrotic gene changes (10), and depletion of langerin-expressing skin-resident DCs worsens chronic skin inflammation and fibrosis in animals (11). A previous study reported that pDCs isolated from the peripheral blood of patients with SSc secreted chemokine CXCL4, and serum CXCL4 levels correlated with skin and lung disease in these patients (12). Additionally, we found increased pDCs in the lungs of patients with SSc (13). However, it is unclear whether pDCs play a direct role in the pathogenesis of systemic fibrosis.

Currently, no treatment reverses fibrosis. Imatinib, a tyrosine kinase inhibitor used to treat leukemia and gastrointestinal tumors (14), inhibits the tyrosine kinase activity of c-abl, c-kit, c-fms, and PDGF receptor 
$(15,16)$. Since c-abl deficiency attenuates the TGF $\beta$-induced extracellular matrix protein production (17) and PDGF plays a role in fibrosis (16), imatinib may target multiple profibrotic pathways in SSc. Indeed, several case series reported the regression of fibrosis following imatinib treatment in patients with graft-versus-host disease and nephrogenic systemic fibrosis. In patients with SSc, treatment with imatinib led to decreased modified Rodnan skin score (mRSS, a measure of skin fibrosis, $P<0.001$ ) and a trend toward improvement of lung functions (18). Similar observations were made in another study, where mRSS decreased by $22.4 \%$ at 12 months $(P=0.001)$, forced vital capacity improved by $6.4 \%$ predicted $(P=0.008)$, and the diffusion capacity remained stable after treatment with imatinib (19). After treatment with low-dose imatinib, $73 \%$ of SSc patients with active pulmonary involvement, unresponsive to cyclophosphamide, had an improved or stabilized lung disease (20). Understanding the effects of imatinib on immune cells may help identify new therapeutic targets for fibrotic diseases.

Here, we detected pDCs in the lymphoid and affected organs in the bleomycin-induced skin and lung fibrosis model. To directly test the role of $\mathrm{pDCs}$ in vivo, we depleted pDCs in bleomycin-injected mice and monitored its effect on skin and lung disease, immune cells, and genes and proteins. In order to translate our findings, we analyzed pDCs in the lung and skin tissues from patients with SSc and control subjects, and in the bronchoalveolar lavage (BAL) and peripheral blood from SSc patients at the baseline and after 1 year of treatment with imatinib. We sought correlation of $\mathrm{pDCs}$ with disease parameters, immune cells, and proteins in patients with SSc. Our results suggest a role of pDCs in the pathogenesis of systemic fibrosis.

\section{Results}

Bleomycin increases $p D C s$ in the affected organs, but not in the spleen, in animals. To test the role of $\mathrm{pDCs}$ in systemic fibrosis in vivo, we used the bleomycin-induced skin and lung fibrosis model. C57BL/6 mice were injected s.c. with bleomycin daily for 14 days, and mediastinal lymph nodes, spleen, and lungs were harvested on day 28. Their single cell suspensions were analyzed for $\mathrm{pDCs}$, identified as live $\mathrm{SSc}^{\text {low }} \mathrm{FSC}^{\text {low }}$ CD19-CD11 ${ }^{\text {int PDCA }-1^{+} \text {B220 }}{ }^{+}$cells. Results show increased pDCs (CD11 $\left.\mathrm{c}^{\text {int/low } P D C A-} 1^{+}\right)$in the lung and lung-draining lymph nodes, but not in the spleen, of bleomycin-injected mice as compared with PBS controls (Figure 1). The observations showing a more profound increase in pDCs, relative to $\mathrm{mDCs}$ $\left(\mathrm{CD} 11 \mathrm{c}^{+} \mathrm{CD} 11 \mathrm{~b}^{+}\right)$, in the lungs and lung-draining lymph nodes but not in the spleen suggest a preferential trafficking of $\mathrm{pDCs}$ to the target organs.

$p D C$ depletion reduces skin and lung fibrosis in the bleomycin model. To determine if $\mathrm{pDC}$ accumulation in the target organs plays a role in the development of fibrosis, we asked if the depletion of pDCs will modulate bleomycin-induced disease. We first demonstrated that a single injection of $100 \mu \mathrm{g}$ of anti-PDCA-1 Ab in naive C57/BL6 mice reduced pDCs but did not affect the frequency of mDCs, B and T cells, and T cell subsets (Supplemental Figure 1). We then established an anti-PDCA-1 Ab regimen that reduces pDCs throughout the course of bleomycin-induced disease. Bleomycin-injected mice were treated with i.p. injections of $100 \mu \mathrm{g}$ of anti-PDCA-1 Ab or isotope-matched control IgG at 2 days before the first bleomycin injection (day -1), and 14 days after the first bleomycin injection (Supplemental Figure 2). With this regimen, pDCs were significantly lower in the lungs on day 7 and day 14, and in lymph nodes and spleen on day 14 and day 28, as compared with control animals.

To assess the clinical effect of pDC depletion, we monitored the treated and control animals for clinical features, including alopecia, submandibular erythema, hunchback appearance, and piloerection (Figure 2A). A clinical severity score comprising these features was estimated for each animal, as described in Methods. As shown in Figure 2B, the clinical severity score was significantly lower in pDC-depleted mice than in isotype control $\mathrm{Ab}$-treated or untreated mice with bleomycin-induced disease. Whereas $50 \%$ animals in both control groups had a score of $\geq 2$, only 1 animal $(5.11 \%)$ in the pDC-depleted group had a score of $\geq 2$ (Figure $2 \mathrm{C}$ ).

Consistent with changes in the clinical score, histopathological changes in the lungs were significantly reduced in pDC-depleted mice as compared with isotype control-treated or untreated bleomycin-injected mice (Figure 2, D and E). Whereas $88 \%$ of control mice had a lung histopathology score of $\geq 2$, only $30 \%$ of $\mathrm{pDC}$-depleted mice had a score of $\geq 2$ (Figure $2 \mathrm{~F}$ ).

Skin fibrosis in these animals was assessed by dermal thickness (Figure 2G) that was significantly lower in pDC-depleted mice than in control Ab-treated or untreated mice with bleomycin-induced fibrosis (Figure $2 \mathrm{H})$. In fact, the dermal thickness in $\mathrm{pDC}$-depleted mice was comparable with that in healthy control mice. 
A

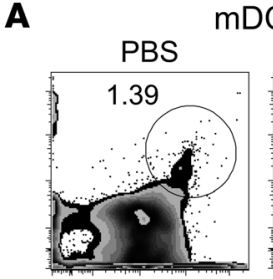

D

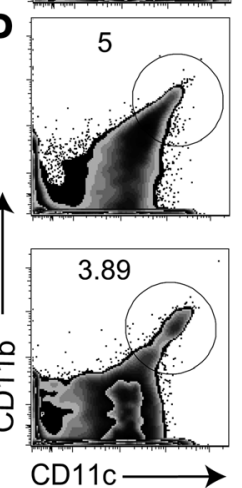

$\mathrm{mDC}$
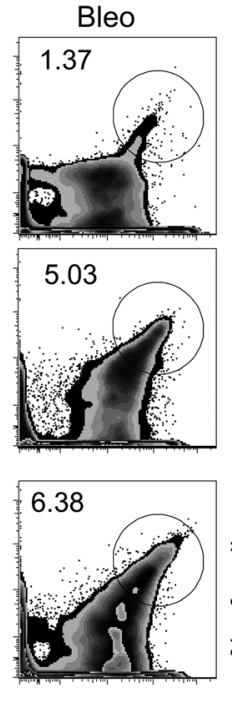

$\mathrm{mDC}$
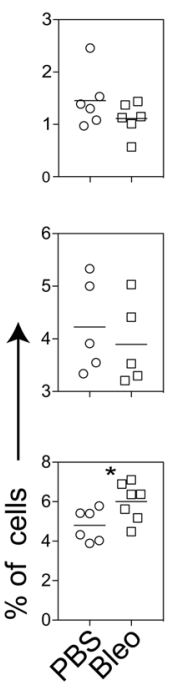

B

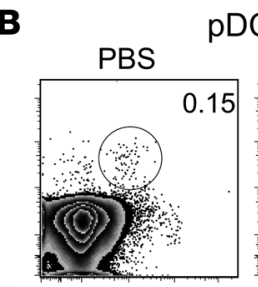

E

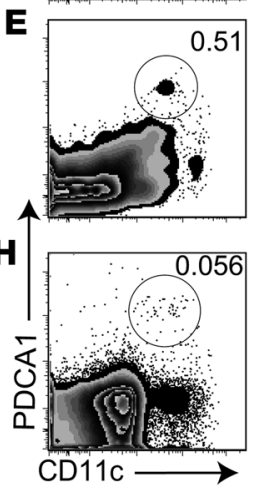

pDC
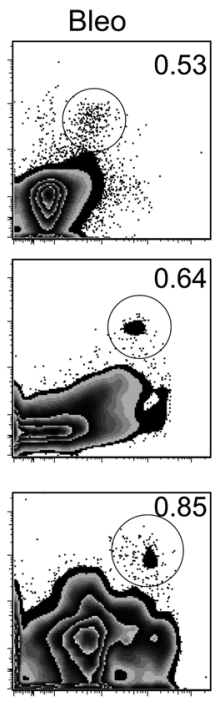

$\mathrm{pDC}$
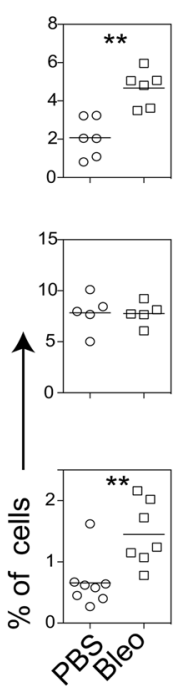

C Relative increase

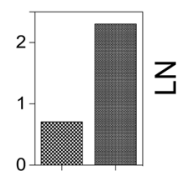

$\mathbf{F}$

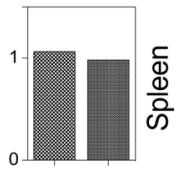

I

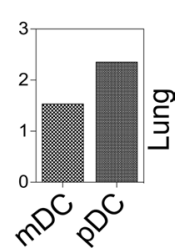

Figure 1. Effect of s.c. bleomycin on DCs in the lung-draining lymph nodes, spleen, and lung. (A, B, D, E, G, and H)Animals were injected s.c. with bleomycin (Bleo) or PBS daily for 2 weeks. Twenty-eight days after the first injection, mediastinal lymph nodes (LN), spleen, and lungs were harvested. Their single cell suspension were analyzed for pDCs and mDCs. Numbers on FACS plots represent the percentage of gated cells: CD11c intPDCA-1+ cells as $\%$ of live

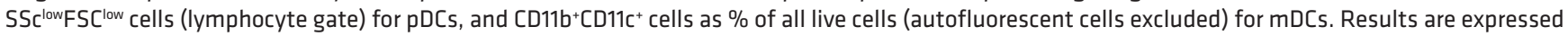
as representative FACS plots and as symbol plots, where each symbol represents a single animal. Horizontal lines denote mean. C, F, and I show a relative increase (ratio) in mDCs and pDCs in bleomycin-injected mice as compared with PBS-injected mice. Results shown are representative of 3 independent experiments. ${ }^{*} P<0.05 ;{ }^{* *} P<0.01$, Mann-Whitney $U$ test.

To assess the effect of pDC depletion on fibrosis, we stained lung and skin tissues with Masson's trichrome and Picrosirius red and measured the collagen content. Trichrome staining (Figure 3, A and B) and Picrosirius red staining imaged in parallel light (Figure 3, C and D) showed reduced collagen deposition in $\mathrm{pDC}$-depleted mice as compared with $\mathrm{pDC}$-intact mice. This was further confirmed by measuring total collagen content using the Sircol assay (Figure 3, G and H). Picrosirius red stain imaged in orthogonal light to display fibrillary collagen assembly demonstrated its decrease in pDC-depleted animals as compared with control animals (Figure 3, E and F). Thus, pDC depletion leads to reduced total collagen and fibrillar collagen in bleomycin-induced skin and lung fibrosis.

To determine whether treatment with anti-PDCA-1 Ab after induction of bleomycin-induced fibrosis is protective, we injected bleomycin s.c. daily from days 1-14 and treated with anti-PDCA-1 Ab on days 7 and 14 (200 $\mu \mathrm{g}$ each) or on days 7, 10, and 14 (100 $\mu \mathrm{g}$ each). The treated animals showed an improvement in skin disease with significantly reduced dermal thickness, but there was no improvement in lung disease (Supplemental Figure 3). However, in these experiments, pDCs were not significantly reduced on day 28 in the lungs and spleen when the treatment was initiated after induction of bleomycin-induced fibrosis (Supplemental Figure 4), although this Ab significantly depleted pDCs when started a day prior to administering bleomycin (Supplemental Figure 2) or when given to naive mice (Supplemental Figure 1). Additional studies are underway to determine the $\mathrm{Ab}$ regimen (dose and route of therapy) that will deplete pDCs that accumulate in large numbers in the lungs of diseased animals.

$p D C$ depletion reduces immune cell infiltrates in the affected organs but not in the spleen. To explore mechanisms underlying pDC's clinical effect, we asked if pDC depletion affects bleomycin-induced immune cell infiltration. On day 28, mDCs were significantly lower in lungs, but not in lymph nodes and spleen, of treated animals compared with controls (Supplemental Figure 2). Total T cells and T cell subsets including NKT, CD4 ${ }^{+}$ and $\mathrm{CD}^{+} \mathrm{T}$ cells, and $\mathrm{B}$ cells were reduced in the lungs of $\mathrm{pDC}$-depleted mice as compared with control $\mathrm{Ab}$-treated bleomycin-injected mice (Figure 4). Total $\mathrm{T}$ and $\mathrm{CD} 4^{+} \mathrm{T}$ cells were also lower in the lung-draining lymph nodes of pDC-depleted mice than controls. In contrast, $\mathrm{T}$ cells and $\mathrm{T}$ cell subsets in the spleen were higher in pDC-depleted mice than in controls. These observations showing reduced immune cells in the affected tissue, along with the higher numbers of these cells in the spleen, upon pDC depletion suggests a possible role of $\mathrm{pDC}$ in regulating the trafficking of immune cells between the lymphoid and affected organs. 
A

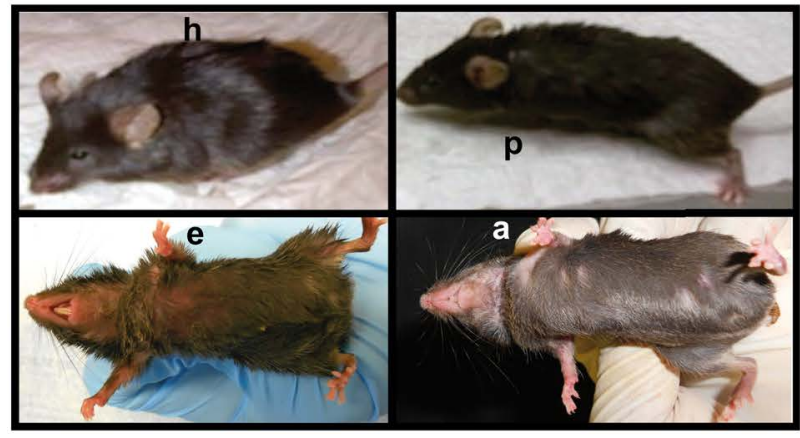

D

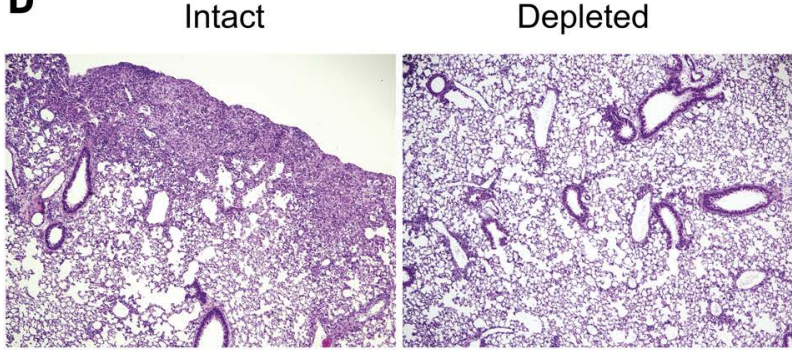

G

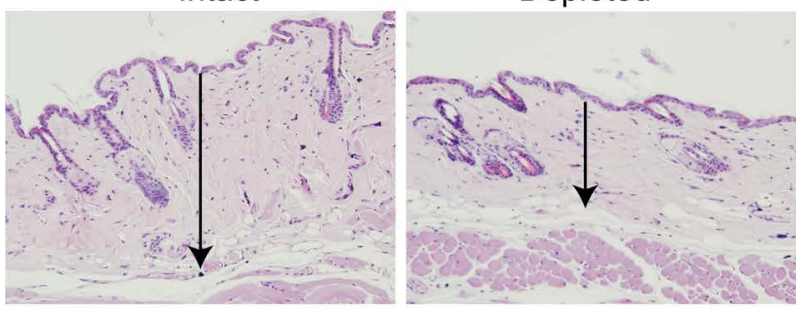

B
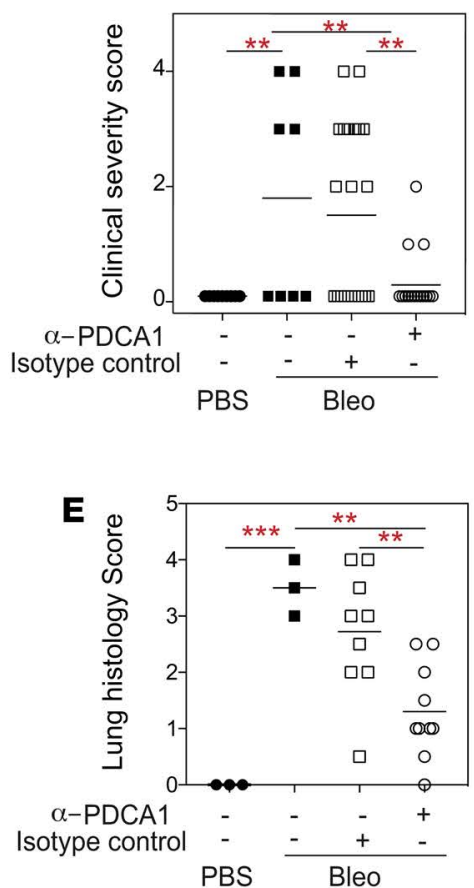

H

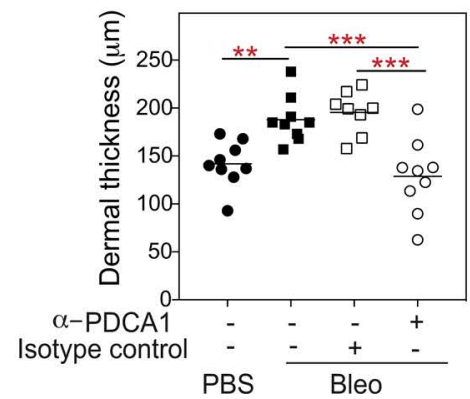

PBS
C
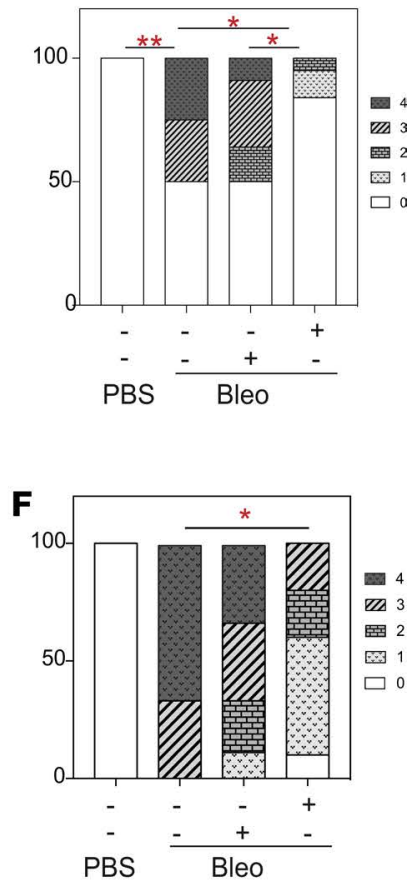

Figure 2. Effect of depletion of pDCs on skin and lung disease in bleomycin-injected animals. Animals were injected with PBS or bleomycin (Bleo), and bleomycin-injected animals treated with PBS, an isotype-matched control Ab (Intact), or an anti-PDCA-1 Ab (Depleted), as described in Supplemental Figure 2A. Animals were monitored for general health and skin lesions, and organs were harvested on day 28. (A) Representative photographs illustrate clinical features, including the hunched back appearance (h), submandibular erythema (e), piloerection (p), and alopecia (a), in bleomycin-injected mice. None of the PBS-injected mice had these lesions. (B and C) Each of the aforementioned features were given a score of 1 , as described in Methods, and clinical severity scores derived for individual animals ( $n=9,9,22$, and 19 animals in PBS, bleomycin alone, bleomycin + control Ab, and bleomycin + anti-PDCA-1 Ab groups, respectively). (D-F) Lung sections stained with H\&E were scored for the severity of lung disease. Representative photomicrographs and histology scores are shown (magnification, 10x). (G and $\mathbf{H}$ ) Skin sections stained with H\&E were analyzed for dermal thickness, as indicated by arrows in the representative photomicrographs (magnification, $4 \times$ ). Results are shown as symbol plots, each symbol representing an animal, and/or as stack plots. Horizontal lines on symbol plots represent mean values. Each stack on stack plots represents proportion of animals with a clinical or histology score, as indicated by the legend on the right of panels. ${ }^{*} P<0.05$, ${ }^{* *} P<0.01,{ }^{* *} P<0.001$ (1-way ANOVA with Newman-Keuls multiple comparisons test in $\mathbf{B}, \mathbf{E}$, and $\mathbf{H} ; \chi^{2}$ test with Yates correction in $\mathbf{C}$ and $\mathbf{F}$ ).

pDC depletion reduces genes and proteins related to chemotaxis, fibrosis, and immune cell activation. Results of a focused gene expression array that includes genes involved in cell migration and activation (Figures 5 and 6) further supports a possible role of $\mathrm{pDCs}$ in the trafficking of inflammatory cells to the affected organs. Genes related to chemotaxis were differentially expressed between bleomycin- and PBS-injected mice ( $\mathrm{Cl} 2$, Ccl3, Ccl4, Ccl7, Ccl8, Ccl11, Ccl12, Cxcl10, and Cxcl12) (Figure 5), and between pDC-depleted and pDC-intact bleomycin-injected mice (Ccl8, $C x c 112, C c r 2, C c r 3, C c r 5, C c r 9, C x c r 1$, and $C x c r 4)$ in the lungs (Figure 6A and Supplemental Figure 5A).

Profibrotic gene $T g f b 1$ that was significantly upregulated (2.76-fold) in bleomycin-injected mice compared with PBS controls had a marked reduction (26.49-fold) in the lungs of pDC-depleted animals compared with $\mathrm{pDC}$-intact controls. In addition, genes related to immune cell activation, inflammation, and 

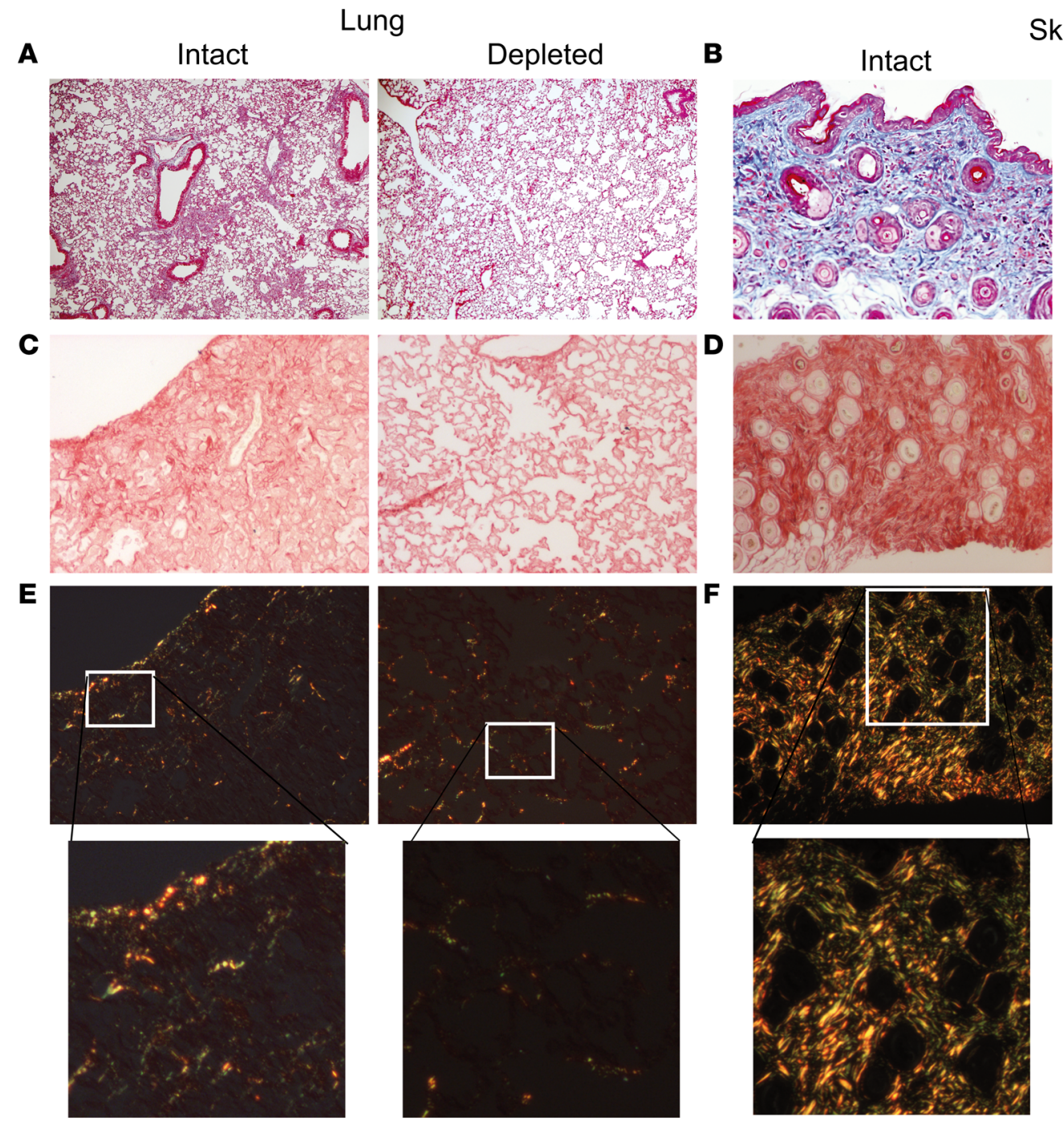

Skin
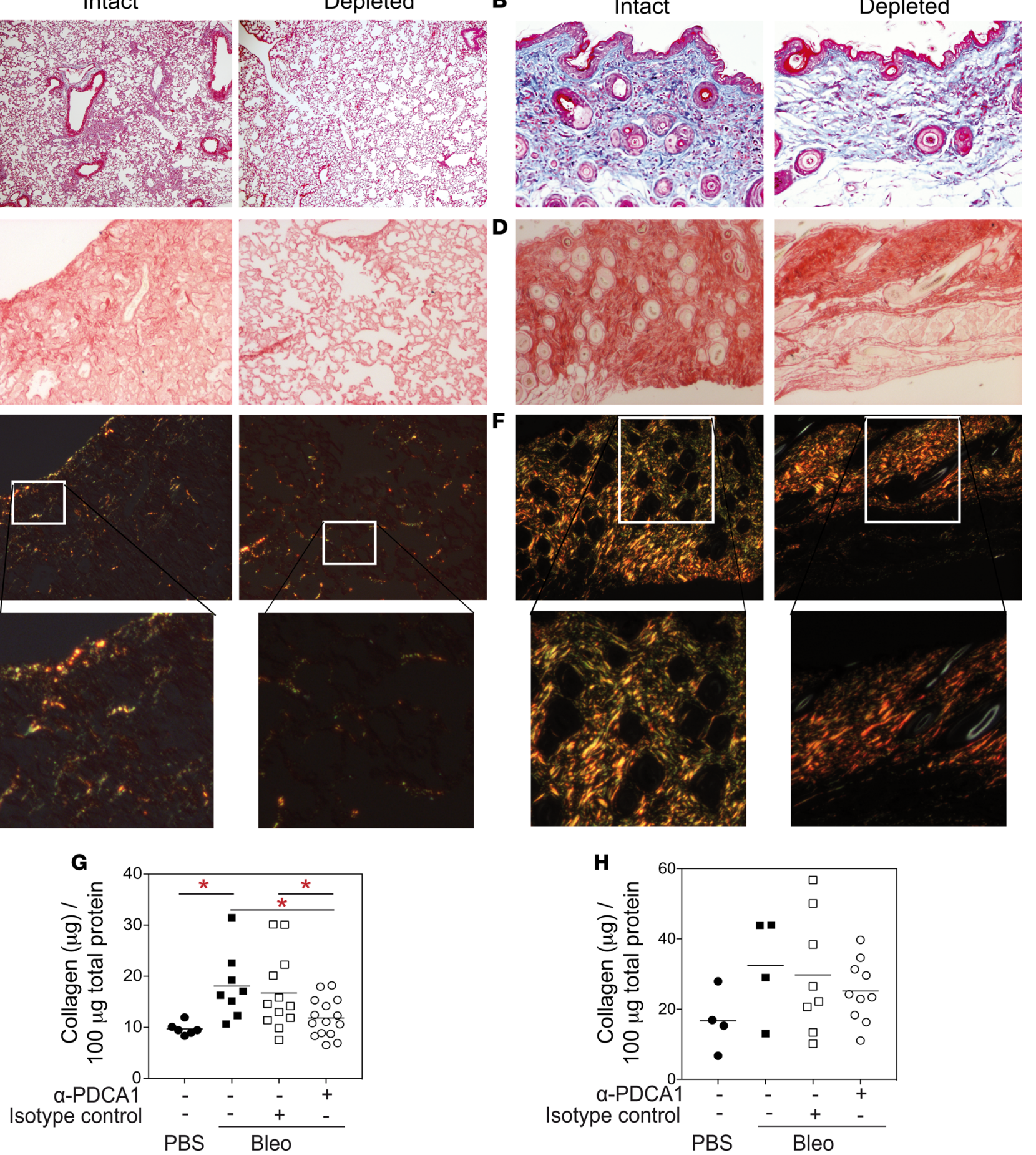

Figure 3. Effect of depletion of pDCs on measures of fibrosis in bleomycin-injected animals. Animals were injected with PBS or bleomycin (Bleo), and bleomycin-injected animals treated with PBS, an isotype-matched control Ab (Intact), or an anti-PDCA-1 Ab (Depleted), as described in Supplemental Figure 2A. Lung (left columns) and skin (right columns) tissues harvested on day 28 were stained with Masson's trichrome (A and B), as well as Picrosirius red stain imaged in parallel light to display total collagen content (C and $\mathbf{D}$ ) or orthogonal light to display fibrillar collagen (E and F). Results are expressed as representative photomicrographs ( $n=$ more than 8 each of intact and depleted animals in $\mathbf{A}$ and $\mathbf{B}$, and 5 each in $\mathbf{C}-\mathbf{F}$ ). Magnification, $10 \times$ for the lung and $4 \times$ for the skin. Photomicrographs below $\mathbf{E}$ and $\mathbf{F}$ show magnified views of insets in these panels. Magnification, $200 \times$ and 20x, respectively. ( $\mathbf{G}$ and $\mathbf{H}$ ) Total soluble collagen content in the lung and skin was measured using the Sircol assay. Results are shown as collagen levels per $100 \mu \mathrm{g}$ of total protein in the tissue. Each symbol represents an animal. Horizontal lines denote mean values. ${ }^{*} P<0.05,1$-way ANOVA with Newman-Keuls multiple comparisons test. 
A

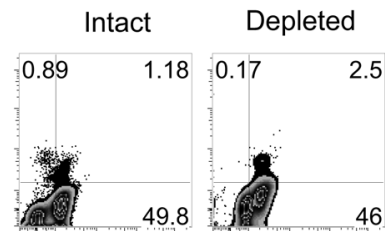

B

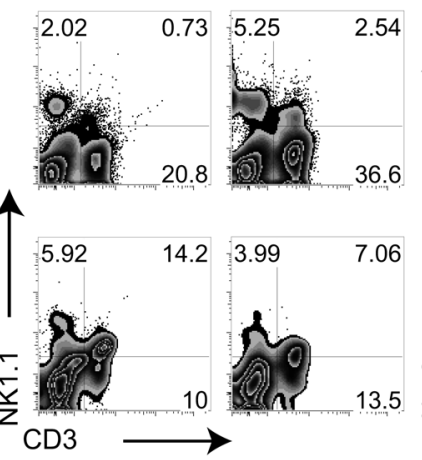

G
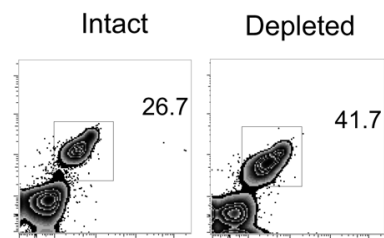

H

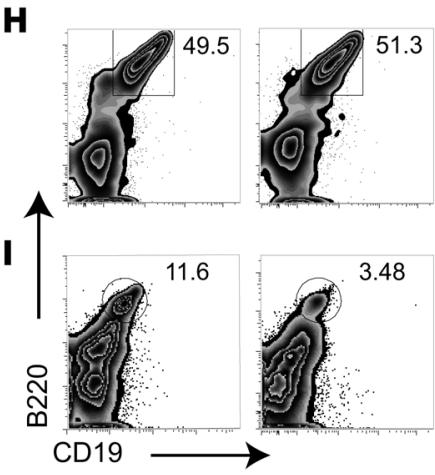

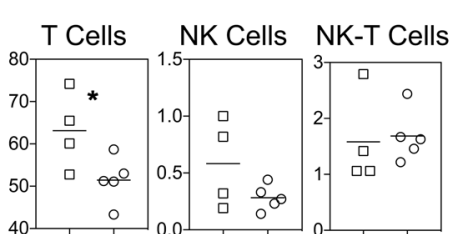

D
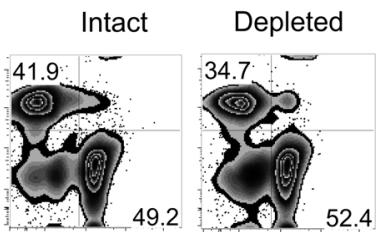

E
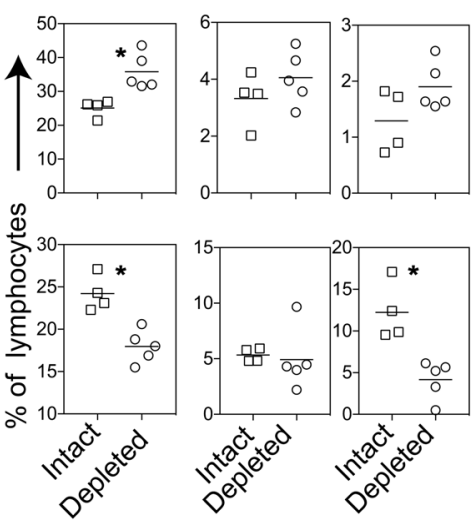

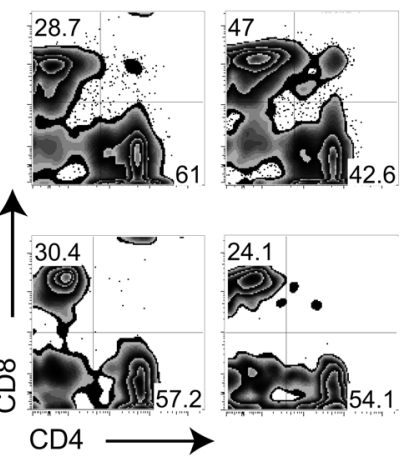

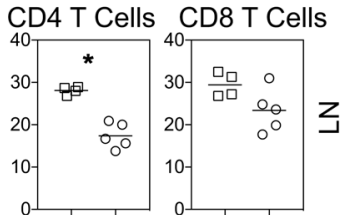
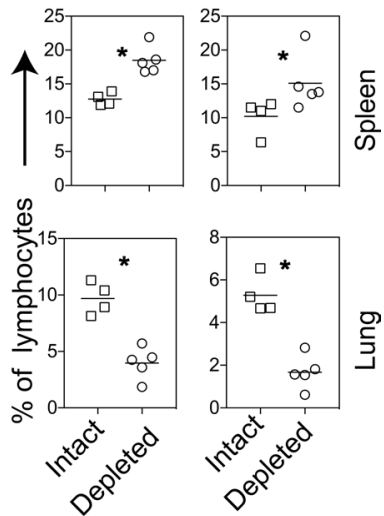

\begin{abstract}
Figure 4. Effect of depletion of pDCs on immune cell subsets in the lung-draining lymph nodes, spleen, and lung. (A-I) Animals were injected with bleomycin and treated with an anti-PDCA-1 Ab (Depleted) or an isotype-matched control Ab (Intact), as described in Supplemental Figure 2A. Lung-draining lymph nodes (LN), spleen, and lungs were harvested on day 28 and analyzed for immune cell subsets. Numbers on representative FACS plots represent the \% of positive cells in the lymphocyte gate; (A-C, and $\mathbf{G}-\mathbf{I})$ or in the TCR $\beta^{+}$cell gate (D-F) dots in symbol plots represent individual animals. Small horizontal lines indicate average values. Results shown are representative of 5 independent experiments, each using 4-5 animals per group. ${ }^{*} P<0.05$, Mann-Whitney $U$ test.
\end{abstract}
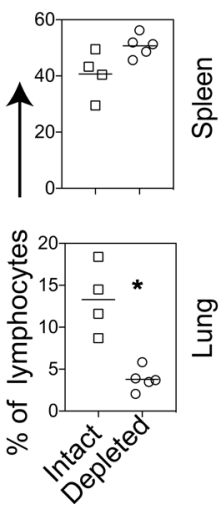

other cellular processes were differentially expressed in the lungs of bleomycin- vs. PBS-injected mice (Figure 5) and of pDC-depleted vs. pDC-intact bleomycin-injected mice (Figure 6A and Supplemental Figure $5 \mathrm{~A})$. Genes implicated in chemotaxis and inflammation were also differentially expressed in the skin between pDC-depleted and intact mice (Figure 6B and Supplemental Figure 5B).

We verified the protein levels of selected differentially expressed genes in the lungs of $\mathrm{pDC}$-intact versus $\mathrm{pDC}$-depleted animals (Figure 7). TGF $\beta 1$ levels in lung extracts and latent TGF $\beta$ on the surface of lung cells were significantly reduced in $\mathrm{pDC}$-depleted mice compared with $\mathrm{pDC}$-intact animals. Levels of thrombospondin-1 (TSP-1) that activates latent TGF $\beta$ (21) were also lower in pDC-depleted mice than in control animals, although the differences were not statistically significant. Levels of most other proteins tested, including CXCL12, CCL2, TLR7, STAT3, CD11b (for Itgam), and B220 (for Ptprc), were complementary to the differentially expressed genes between pDC-depleted and control animals.

$p D C$ s are reduced in the peripheral blood but increase at the disease site in patients with SSc. To translate the above animal model findings, we analyzed DCs in the peripheral blood of patients with SSc and sex/ race/ethnicity/age-matched ( \pm 5 years) healthy donors (Supplemental Table 1 ). The proportions and absolute number of circulating pDCs, defined as $\mathrm{CD} 123^{+} \mathrm{CD} 11 \mathrm{c}-\mathrm{HLA}-\mathrm{DR}{ }^{+} \mathrm{BDCA}-2^{+}$cells, were lower in patients with SSc than in heathy donors (Figure 8, A and B), whereas the proportions and number of 
A

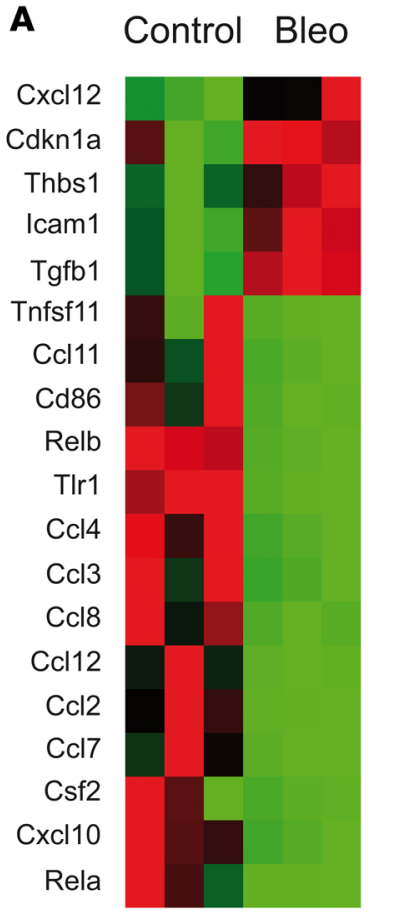

Fold

regulation

2.51

3.04

2.52

5.03

2.76

$-26.78$

$-5.91$

$-4.56$

$-22.17$

$-9.75$

$-8.21$

$-5.40$

$-3.61$

$-11.38$

$-11.35$

$-14.61$

$-15.92$

$-5.11$

$-7.52$

Magnitude of gene expression

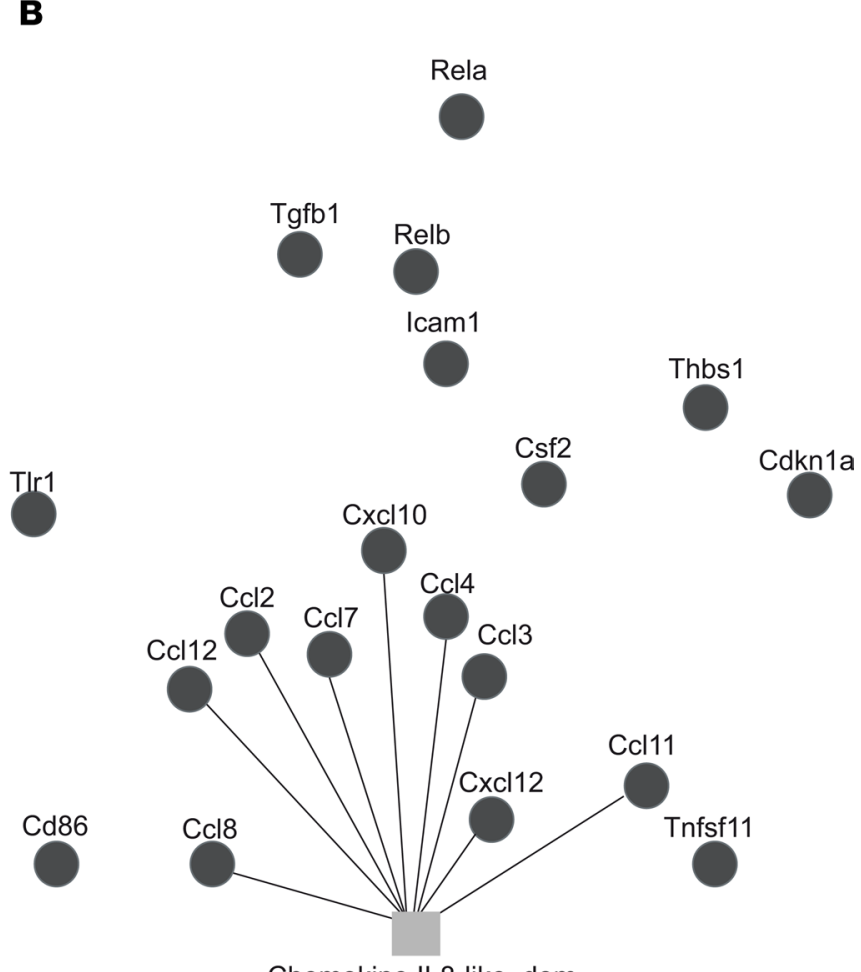

Chemokine IL8-like_dom

Figure 5. Effect of bleomycin on the expression of genes related to DC and antigen presenting cell function. Lung tissues from PBS- (Control) and bleomycin-injected (Bleo) mice were profiled using the Mouse Dendritic and Antigen Presenting Cell RT² Profiler PCR Array (Supplemental Figure 11). Fold changes were calculated and expressed as the log-normalized ratios of the bleomycin/control groups. For multiple comparisons, we applied a FDR approach using 2-stage linear step-up procedure of Benjamini, Krieger, and Yekutieli, with a $q$ of $15 \%$. Results of differentially expressed genes with their $P$ values, $q$ values, and fold-changes are shown. ${ }^{*} P<0.05,{ }^{* *} P<0.01,{ }^{* * *} P<0.0001$ (A). (B) Gene function network analysis of differentially expressed genes was performed using GeneMANIA (51) (Supplemental Figure 5).

circulating $\mathrm{CD} 123^{-} \mathrm{CD} 11 \mathrm{c}^{+}$cells (mDC/non-pDC) were not significantly different between patients and heathy donors (Supplemental Figure 6).

To analyze pDCs at the disease site, we collected BAL samples at the baseline from 15 patients who were enrolled in a clinical trial of imatinib for treatment of SSc. Patients had high-resolution CT (HRCT) scans of lungs performed to assess the severity of lung fibrosis (Supplemental Figure 7). Since the disease (HRCT) scores were substantially different between right middle lobe (RML) and right lower lobe (RLL) (Supplemental Table 2), RML and RLL BAL samples were analyzed separately for all but the first 2 patients. BAL samples from patients who had bronchoscopy for respiratory symptoms but were found to have no significant lung disease on further diagnostic evaluations were used as controls. While only $10 \%-15 \%$ of $\mathrm{CD} 11 \mathrm{c}^{-} \mathrm{CD} 123^{+}$cells expressed other $\mathrm{pDC}$ markers (BDCA-2 and HLA-DR) in the peripheral blood (Figure 8A), 90\%-98\% of CD11 $\mathrm{c}^{-} \mathrm{CD} 123^{+}$cells were HLA-DR ${ }^{+} \mathrm{BDCA}-2^{+}$in $\mathrm{BAL}$ from patients with SSc (Figure 8C), suggesting a preferential accumulation of pDCs among myeloid cells that express CD123 to the lungs in patients with SSc-associated interstitial lung disease (ILD). As compared with controls, BAL samples from SSc patients had significantly increased pDCs (Figure 8D).

To confirm that pDCs infiltrate the affected organs, we stained lung tissues from SSc patients and nonSSc controls to detect pDCs. While few pDCs were detected in the control lung tissues, SSc lungs had increased pDCs in the interstitial tissue and bronchi (Figure 8E). Similarly, skin biopsies showed more pDCs in SSc patients than in healthy controls (Figure 8F). Thus, pDCs are decreased in the circulation but are increased in the affected organs. These observations suggest a possible trafficking of pDCs to the disease site.

$p D C$ s in BAL correlate with disease severity in patients with SSc. To explore a possible link between $\mathrm{pDC}$ accumulation at the disease site and disease development, HRCT scans of lungs from SSc patients enrolled 


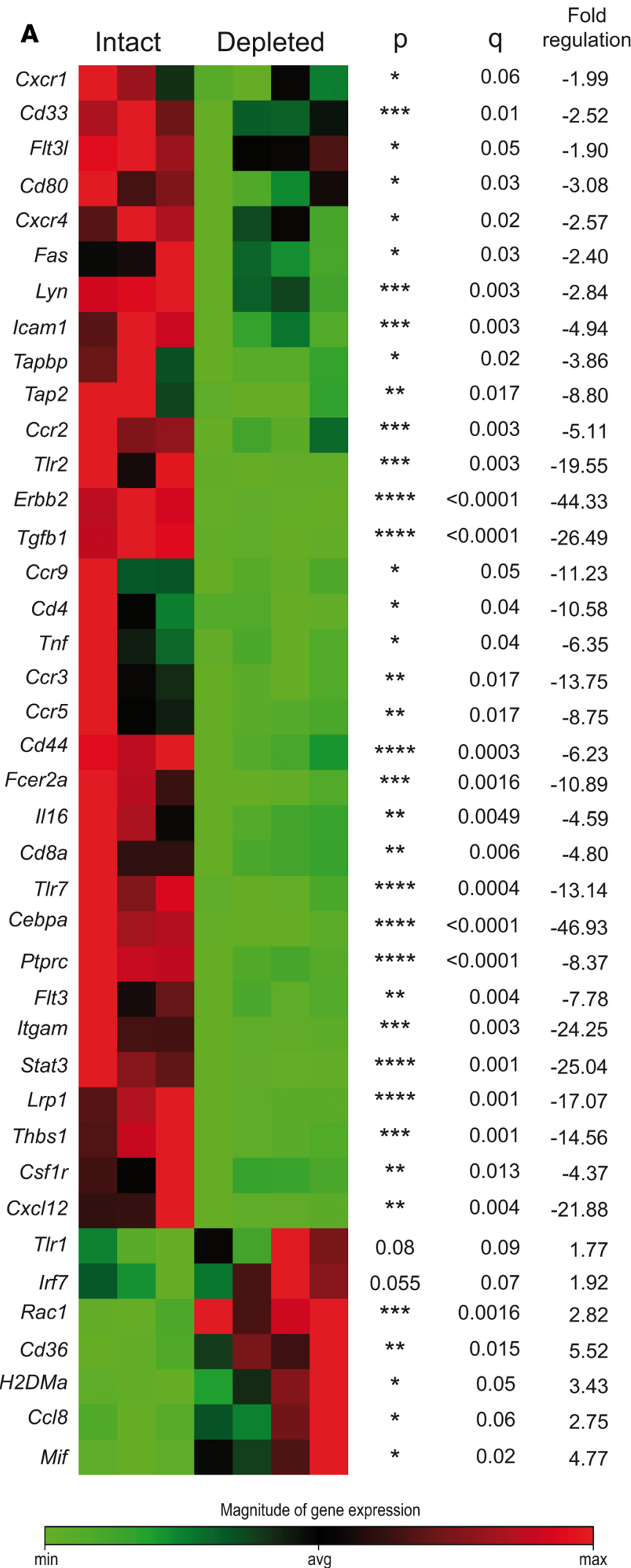

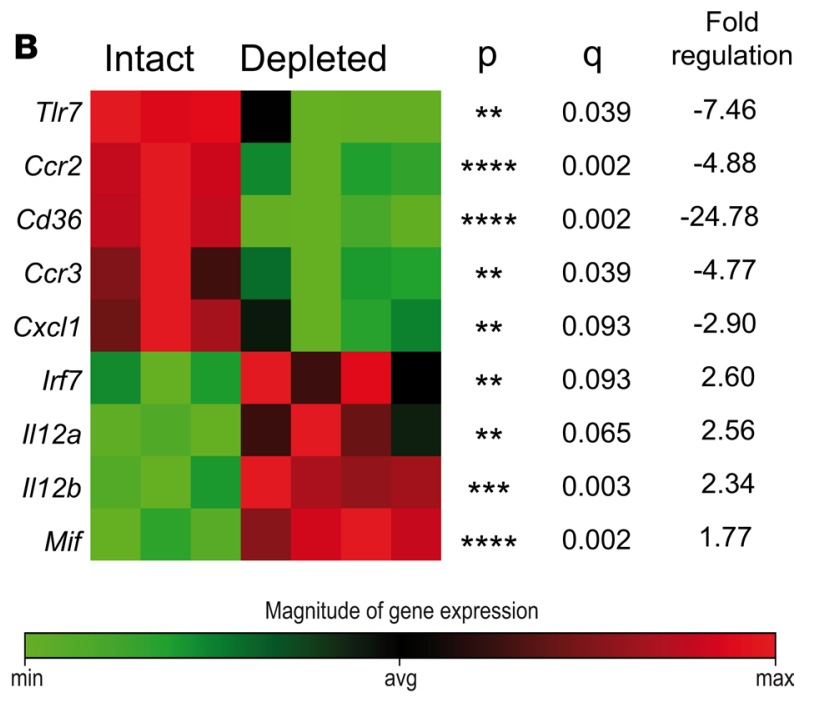

Figure 6. Effect of pDC depletion on the expression of genes related to $D C$ and antigen presenting cell function in bleomycin-injected mice. Lung (A) and skin (B) tissues from pDC-depleted and intact bleomycin-injected mice were profiled using the Mouse Dendritic and Antigen Presenting Cell $\mathrm{RT}^{2}$ Profiler PCR Array (Supplemental Figure 12). Multiple 2-tailed $t$ test and FDR analysis was performed using the corrected method of Benjamini and Yekutieli with a desired FDR (q) of $10 \%$. Fold changes of the differentially expressed genes and $P$ and $q$ values are presented on the right of heatmaps. In the lungs, 40 genes were differentially expressed between pDC-intact and -depleted mice, of which 33 genes were significantly reduced and 7 were increased in pDC-depleted animals as compared with pDC-intact animals. In the skin, 9 genes were differentially expressed between $\mathrm{pDC}$-intact and -depleted mice, of which 5 genes were significantly reduced and 4 genes were increased in pDC-depleted animals as compared with pDC-intact mice. Results represent 2 experiments. ${ }^{*} P<0.05 ;{ }^{* *} P<0.01,{ }^{* * *} P<0.001,{ }^{* * * *} P<0.0001(n=4$ pDC-depleted and 3 intact mice). 
A

TGFß1

B

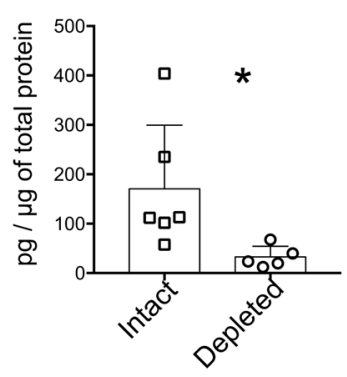

TLR7
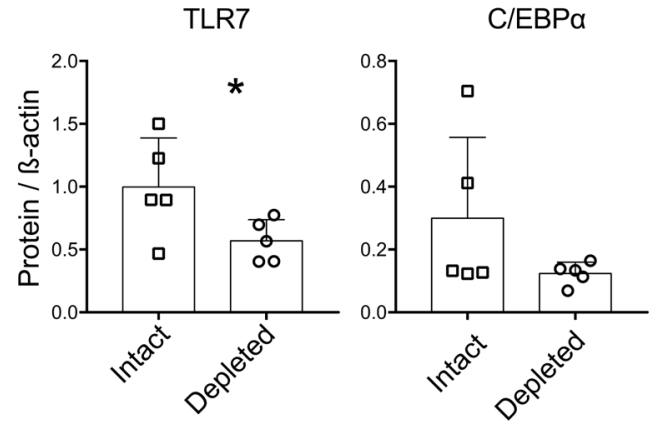

TSP-1

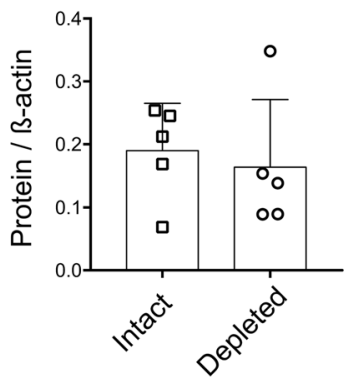

C

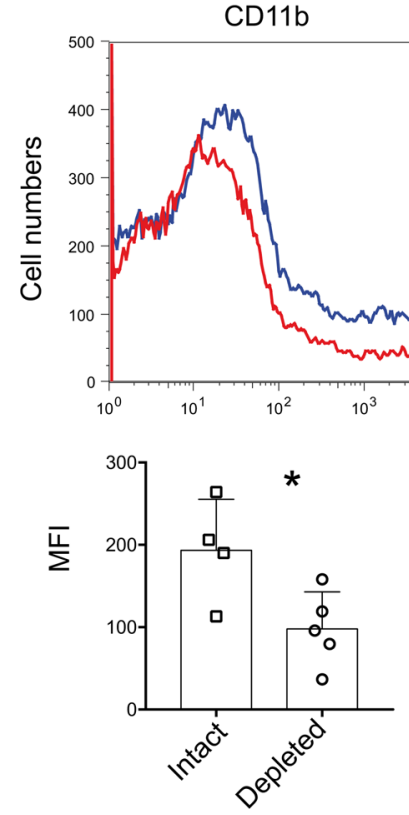

CXCL12

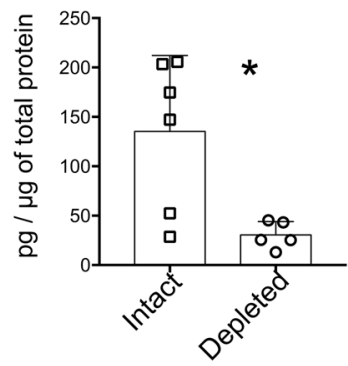

B220

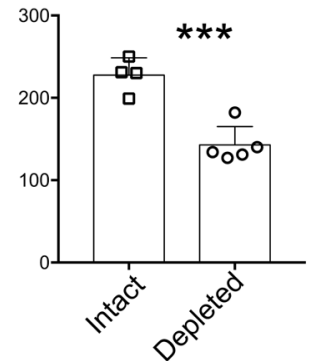

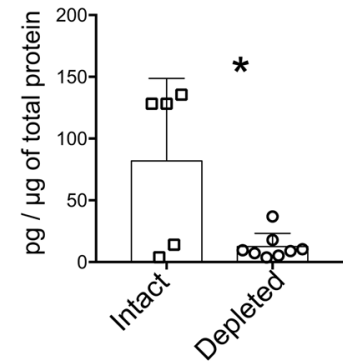

STAT3

Rac-1
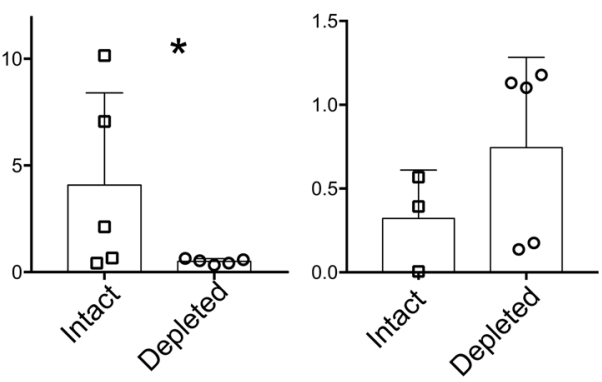

LRP1
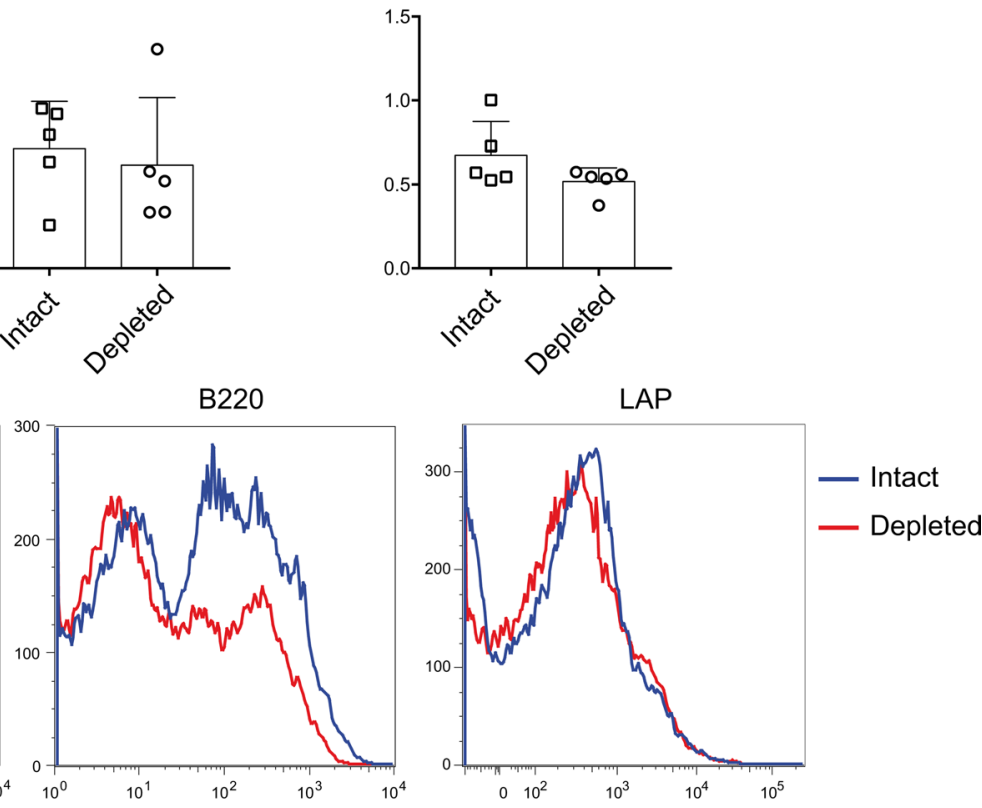

Figure 7. Effect of pDC depletion on protein levels for selected differentially expressed genes in bleomycin-injected mice. Protein extracts or freshly isolated cells from the lungs of pDC-depleted and-intact bleomycin-injected mice were profiled for proteins for 10 downregulated genes and 1 upregulated gene (Rac1), as shown in Figure 6. (A) Levels of TCF 31, CXCL12, and CCR2 ligand (CCL2) were measured by ELISA. (B) Expression levels of TLR7, C/ EBP $\alpha$, STAT3, Rac-1, thrombospondin-1 (TSP-1, Thbs1 gene), and LRP1 were assayed by Western blot. (C) Surface expression of CD11b (for Itgam), B220 
(for Ptprc), and LAP (latency associated peptide) for latent TGF $\beta$ was detected by flow cytometry. Results are expressed as representative Western blot and flow cytometry plots, symbol plots with each symbol representing an animal, and as the mean \pm SEM values. MFI, mean fluorescent intensity. ${ }^{*} P<$ $0.05,{ }^{* *} P<0.001$ (unpaired 2-tailed $t$ test).

in the imatinib trial were scored (Supplemental Figure 7) for disease severity parameters, including ground glass opacity (GGO) and fibrosis prior to starting the medication (Supplemental Table 2). The frequency of pDCs in BAL correlated with both GGO and fibrosis scores at the baseline (Figure 9A) but more strongly associated with GGO that is believed to represent the early stage of SSc-ILD $(22,23)$. Non-pDCs/mDCs (CD123-CD11 $\mathrm{c}^{+}$cells) did not correlate with GGO. We then compared $\mathrm{pDC}$ frequencies between patients with $<20 \%$ vs. $\geq 20 \%$ HRCT scores, since a $\geq 20 \%-25 \%$ extent of SSc-ILD has been reported as an independent predictor of disease progression including mortality in SSc $(24,25)$. The frequency of pDC in BAL, but not in blood, could discriminate patients based on 20\% GGO cut-off (Figure 9B). pDCs also negatively correlated with disease duration (Figure 9C). These observations suggest a possible role of pDCs during early stages of SSc.

$B A L$ pDCs correlate with local immune cell infiltrates and cytokines in patients with SSc. To assess a link between pDCs and inflammatory cell infiltrate in the affected organs, we sought a correlation between the frequency of pDCs and of various immune cells and their cytokine responses. BAL pDCs correlated with the frequency of $\mathrm{CD}^{+}{ }^{+} \mathrm{T}$ cells and of IL-4-producing T cells in the BAL (Figure 9, D and E) but not with the frequency of $\mathrm{CD} 8^{+} \mathrm{T}$ cells or IL-17- or IFN $\gamma$-producing cells in the BAL. Furthermore, staining of consecutive sections of skin and lung biopsies from SSc patients showed a colocalization of BDCA-2 and TGF $\beta 1$ (Supplemental Figure 8), suggesting a possible role of pDCs in producing profibrotic cytokine TGF $\beta$. Thus, in patients with SSc, pDCs at the affected site associate with cytokines IL-4 and TGF $\beta$, which have been implicated in the development of fibrosis.

$p D C$ s in the BAL are reduced after treatment with imatinib in patients with SSc. We and others have reported that treatment with imatinib led to the improved $\operatorname{mRSS}(18,26)$ and a stabilization or trend toward improvement in $\operatorname{ILD}(18,20)$. Previous studies have shown that imatinib inhibits the tyrosine kinase activity of c-fms (15) that is required for macrophage CSF (M-CSF) to promote pDC development (27). Therefore, we asked if imatinib treatment affects pDC infiltration in lungs. As shown in Figure 10, pDC frequencies were reduced in the BAL, but not in the peripheral blood, of patients with SSc after 1 year of treatment with imatinib. Additionally, changes in the frequency of BAL pDCs between baseline and after imatinib $(\Delta \mathrm{pDC})$ correlated with the changes in fibrosis scores $(r=-0.62)$. Thus, pDCs were significantly reduced in the affected tissue, but not in circulation, of patients after treatment with imatinib, which is known to reduce fibrosis. Additionally, decreased pDCs in BAL correlated with improved/stable SSc-ILD after treatment.

\section{Discussion}

This article demonstrates the accumulation of pDCs in the organs affected by fibrosis in animals and humans. The depletion of pDCs reduced fibrosis, immune cell infiltration, and the expression of genes and proteins involved in fibrosis, chemotaxis, inflammation, and DC differentiation in the skin and lung of animals. In resonance with the animal model findings, pDCs in lungs of patients with SSc correlated with the severity of lung disease - and with local $\mathrm{T}$ cell infiltration — and associated with profibrotic cytokines (IL-4 and TGF $\beta$ ). Furthermore, treatment with imatinib that has been shown to improve SSc skin and lung disease and/or prevent its deterioration $(18-20,26)$ reduced pDCs in the lungs of patients with SSc. Taken together, these observations suggest a potential role of $\mathrm{pDC}$ accumulation at the affected organs in the pathogenesis of systemic fibrosis.

Not all DCs are pathogenic in SSc. While pDCs correlated with the severity of lung disease in patients and $\mathrm{pDC}$ depletion in animals reduced fibrosis, $\mathrm{mDC}$ /non-pDCs did not correlate with ILD severity in SSc patients. In a previous report, depletion of non-pDCs worsened bleomycin-induced profibrotic gene changes such as TGF $\beta 1$-induced upregulation of connective tissue growth factor $(C \operatorname{tg} f)$ in the skin (10). Further analysis of different DC subsets at different stages of SSc disease may unearth diverse roles of DC subsets in fibrosis development.

In both animals and humans, we found a preferential increase in pDCs in the affected organs (skin/ lungs), but not systemically (spleen/blood). In fact, peripheral blood pDCs were lower in patients with SSc than in healthy volunteers. These observations suggest a trafficking of pDCs to the affected organs and/or their local activation during the development of fibrosis. When we analyzed the expression of 
A

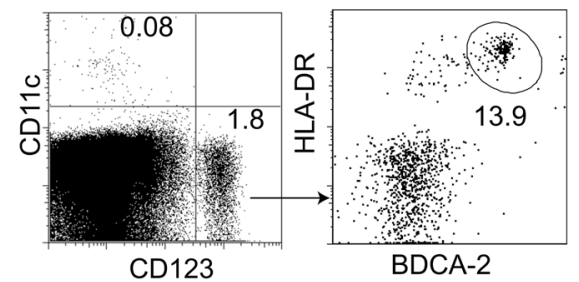

C

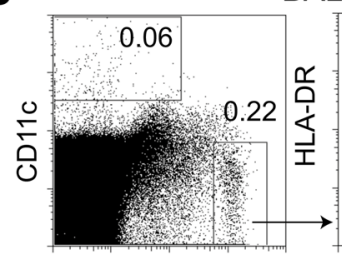

CD123

E

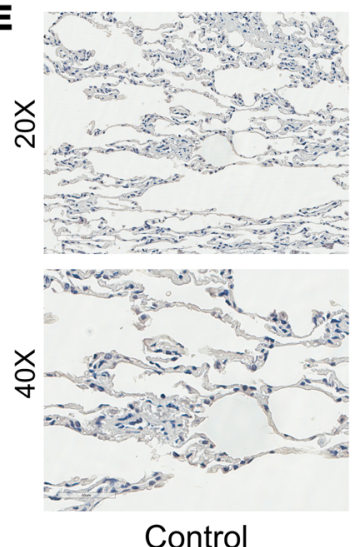

Control
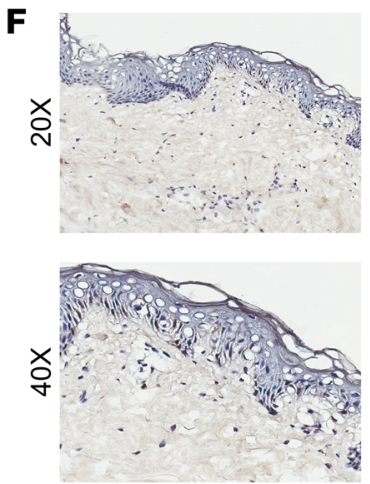

Control
BAL

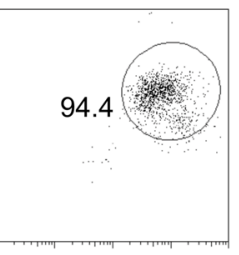

BDCA-2
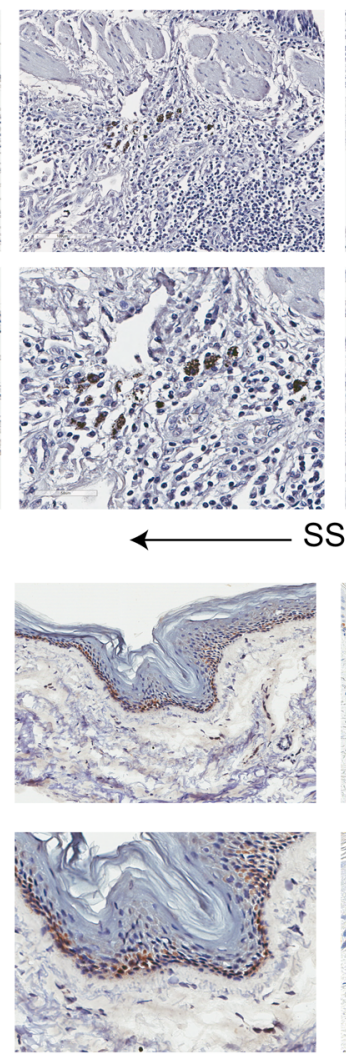

SSc

D
B
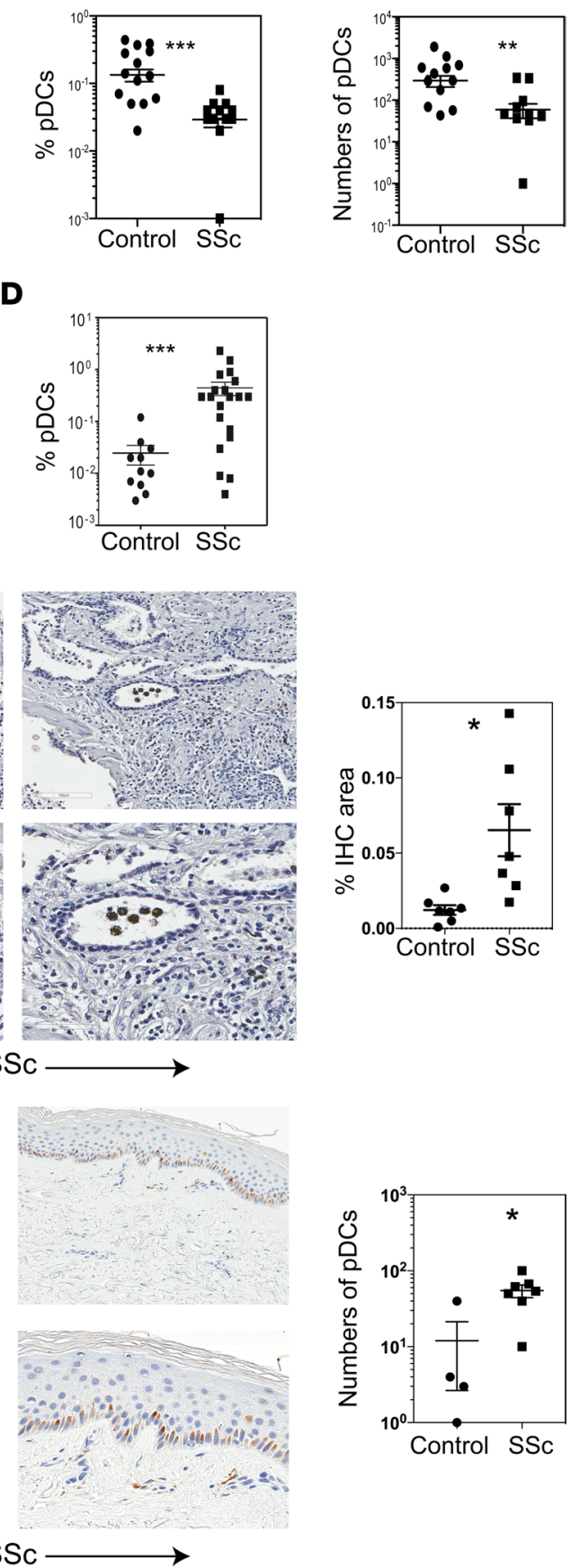

Figure 8. Detection of pDCs in the peripheral blood, BAL, and lung and skin tissues from patients with SSc and controls. (A and B) Peripheral blood mononuclear cells from 15 each of healthy controls and patients with SSc were analyzed by flow cytometry for CD11c, CD123, HLA-DR, and BDCA-2 expression. Representative dot plots show $C D 11 c^{-} C D 123^{+}$cells that were further analyzed for $\mathrm{HLA}-\mathrm{DR} \mathrm{R}^{+} \mathrm{BDCA}-2^{+}$cells, which are expressed as $\%$ of gated CD11c ${ }^{-}$CD123+ cells on dot plots (A). The percentage (expressed as the \% of CD123+CD11c ${ }^{-}$HLA-DR ${ }^{+} B D C A-2^{+}$cells of total blood cells) and total number of peripheral blood pDCs were compared between healthy controls and SSc patients (B). Data are shown as individual sample values and as the mean \pm SEM. ${ }^{* *} P<0.01$ and ${ }^{* * *} P<0.001$ (unpaired 2-tailed $t$ test). (C and $\left.\mathbf{D}\right)$ BAL cells from SSc-ILD patients before enrollment into the imatinib trial were analyzed by flow cytometry for CD11c, CD123, HLA-DR, and BDCA-2. Representative dot plots from SSc patients show CD11c CD123+ cells that were further analyzed for $\mathrm{HLA}-\mathrm{DR}{ }^{+} \mathrm{BDCA}-2^{+}$cells (C). BAL samples from patients who had bronchoscopy for respiratory symptoms but not found to have any significant lung disease on further evaluations were used as controls. The percentage of BAL pDCs were compared between SSc-ILD patients and controls (D). pDCs are expressed as the $\%$ of CD123+CD11c-HLA-DR ${ }^{+} B D C A-2^{+}$cells of total BAL cells (mean \pm SEM; ${ }^{* * *} P$ $<0.001$, unpaired t-test). (E) Paraffin-embedded sections $(4 \mu \mathrm{m})$ of lung tissues were obtained from SSc-lung explants and control lungs (wedge resection for spontaneous pneumothorax or traumatic lung laceration or lungs collected at autopsy from cases with renal cancer or liver cirrhosis). The sections were stained with anti-BDCA-2 Ab to detect pDCs. While the control lungs had no to a few BDCA-2+ cells (left panels), all SSc lungs show BDCA-2+ cells in the interstitial tissue (middle and right panels) and bronchi (right panels). Stained sections were digitized using the Aperio scanning system. Results from individual subjects are expressed in a symbol plot as the percent IHC-stained area (mean \pm SEM; ${ }^{*} P=0.01$, unpaired 
2-tailed $t$ test). (F) Frozen sections $(6 \mu \mathrm{m})$ of 6 -mm punch skin biopsies from patients with 4 SSc and 2 healthy volunteers were stained for BDCA-2. Similar results were obtained in a separate experiment using paraffin-embedded skin sections from 4 each of SSc patients and healthy volunteers. Representative photomicrographs show no to rare BDCA-2+ cells in control skin (left panels), but abundant BDCA-2+ cells in the epidermis and dermis of 2 SSc patients: a 41-year-old man with an mRSS of 33 (middle panels) and a 21-year-old woman with an mRSS of 25 (right panels). The stained cells were manually counted, and the results from individual subjects are shown in a symbol plot as the number of pDCs per mm ${ }^{3}$ (mean \pm SEM; ${ }^{*} P=0.01$, unpaired 2 -tailed $t$ test).

genes that could be involved in DC trafficking and local activation, our results showed that Cxcl12, which is known to regulate pDC migration $(28,29)$, was increased in the lungs of bleomycin-injected mice and that both $C x c l 12$ and its receptor $C x c r 4$ were reduced by 22 -fold and 2.6-fold, respectively, in pDC-depleted mice. CXCL12 protein levels were also reduced in the lungs of pDC-depleted animals. CCR5 that drives $\mathrm{pDC}$ migration to inflamed tissues and CCR9 that is required for $\mathrm{pDC}$ migration to the intestine (28) were decreased in the lungs of pDC-depleted mice. CCR2 drives recruitment of pDCs to the skin upon TLR7 activation (28); both $C \mathrm{cr} 2$ and $T l r 7$ were decreased by 5- to 13 -fold in the skin and lungs of pDC-depleted mice. Protein levels of CCR2 ligand (CCL2) were also reduced in the lungs of pDC-depleted mice. FLT3 and its ligand (FLT3L) promote pDC development through activation of STAT3 (28); the gene expression levels of all 3 (Flt3, Flt3l, and Stat3) were significantly reduced in pDC-depleted animals. Transcription factor Cebpa that plays a role in early-state DC differentiation (30) and tyrosine kinase Erbb2 that can enhance the maturation and function of DC (31) were 2 of the most downregulated genes ( $>40$-fold) in the lungs of pDC-depleted mice.

In humans, $\mathrm{pDC}$ frequencies in BAL correlated with local levels of proteins involved in chemotaxis and leukocyte migration (CCL24, IL-16, PAI1) in BAL fluid at the baseline (Supplemental Figure 9). In addition, we found a correlation between BAL pDC frequency and local levels of IL-3 and M-CSF, which can promote $\mathrm{pDC}$ survival and differentiation $(27,32,33)$. Thus, both increased trafficking and local differentiation and survival might contribute to $\mathrm{pDC}$ accumulation at the affected sites. Ongoing studies will determine the cellular source of the differentially expressed molecules and their relative contribution to pDC accumulation in affected organs.

Multiple mechanisms may underlie the ability of $\mathrm{pDC}$ to promote the development and progression of fibrosis. First, pDC depletion in animals reduced infiltration with T cells, NKT cells, and B cells in the affected organs, but not in the spleen, with a reduced expression of genes implicated in chemotaxis in lung and skin. In resonance with the animal findings, BAL pDC frequency in SSc patients correlated with local $\mathrm{CD}^{+} \mathrm{T}$ cells, and both correlated with CCL24 (Supplemental Figure 9) (4), which is a known chemoattractant for $\mathrm{CD}^{+} \mathrm{T}$ cells (34). Additionally, BAL pDCs correlated with the local levels of IL-16, which is also known to promote the recruitment of $\mathrm{CD}^{+} \mathrm{T}$ cells into tissues (35). BAL pDCs correlated with GGO, which represents earlier stages of $\operatorname{SSc}-\operatorname{ILD}(22,23)$, and negatively correlated with disease duration. Taken together, these observations suggest a possible role of $\mathrm{pDCs}$ in regulating the trafficking of lymphocytes to target organs during the earlier stages of development of systemic fibrosis.

Second, BAL pDCs correlated with IL-4-producing T cells at the baseline. After imatinib, pDCs were reduced (Figure 10), as were IL-4-producing T cells (4). Since type 2 cytokines play a role in the development of fibrosis $(4,36,37)$, pDCs might contribute to SSc pathogenesis by inducing type 2 cytokine responses. We found that BAL pDC frequencies correlated with IL-3 levels in BAL of patients with SSc (Supplemental Figure 9). Since IL-3-stimulated pDCs can induce Th2 differentiation $(33,38)$, increased IL-3 in BAL of SSc patients may be a potential mechanism of increased IL-4-producing T cells in SSc lungs.

Third, pDC depletion resulted in a 26-fold reduction in Tgfb1 gene expression, a significant decrease in TGF $\beta 1$ protein levels, and a significantly decreased latent TGF $\beta$ peptide on the cell surface in the lungs of animals. Furthermore, staining of consecutive sections of skin and lung biopsies from SSc patients suggested a colocalization of BDCA-2 and TGF $\beta 1$ (Supplemental Figure 8). pDC-depleted animals also had a 25 -fold reduction in Stat3 gene expression and a significant decrease in STAT3 protein that is being investigated as a therapeutic target for fibrosis (39). Additionally, levels of profibrotic proteins, including PAI1-a, PDGF-AB, and IL-9, in human BAL correlated with local pDC frequency at the baseline (Supplemental Figure 9). These observations suggest a potential role of $\mathrm{pDCs}$ in producing/inducing profibrotic cytokine(s). Thus, modulation of chemotaxis of inflammatory cells, induction of type 2 cytokines, and induction/production of profibrotic cytokines such as TGF $\beta 1$ may underlie the ability of pDCs to promote the development and progression of fibrosis. 
A

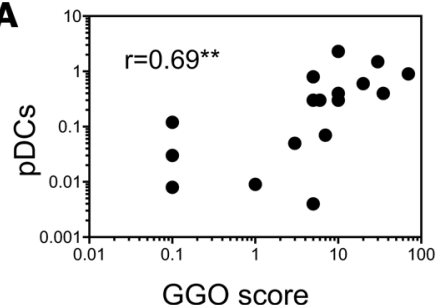

B

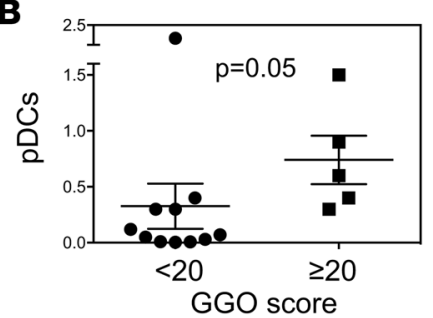

D

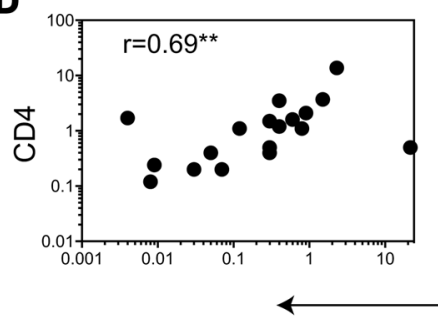

$\mathbf{E}$

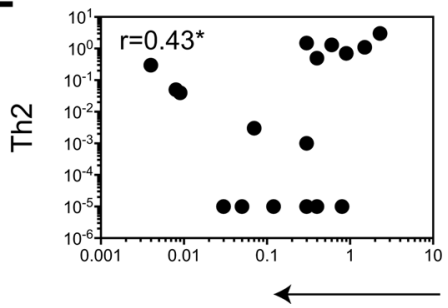

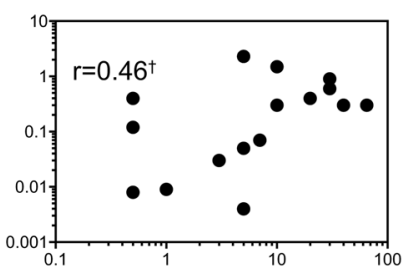

Fibrosis score

C
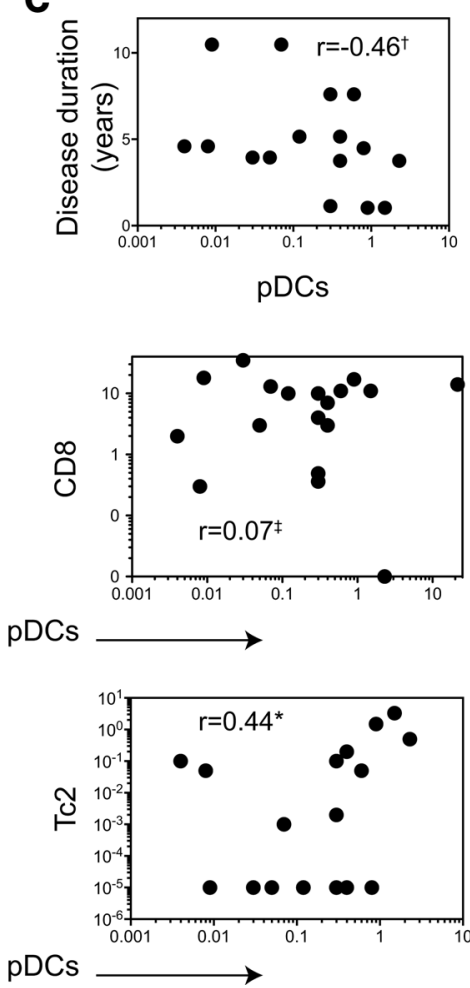

Figure 9. Correlation between disease parameters and pDCs in patients with SSc. The frequencies of pDCs in BAL samples were correlated with: (A) HRCT scores of GGO and fibrosis, (C) with disease duration of SSc, (D) with the frequencies of T cell subsets, and $(\mathbf{E})$ with the frequencies of IL-4-producing $\mathrm{CD}^{+}$and $\mathrm{CD4} 4^{-}$cells (referred to as Th2 and Tc2, respectively) in the BAL from SSc patients. Cell subsets are expressed as the frequencies of total cells in the BAL. $r$ values from nonparametric Spearman correlations are indicated on plots. ${ }^{*} P<0.01,{ }^{*} P<0.05,{ }^{\dagger} P=0.05-0.09$, and ${ }^{\ddagger} P>0$.1. (B) The pDC frequencies in SSc patients with low $(<20 \%, n=13)$ vs. high ( $\geq 20 \%, n=5)$ GGO scores (mean \pm SEM; $P=$ 0.05 , Mann-Whitney $U$ test).

In a previous report, patients with chronic myeloid leukemia who were in complete response after imatinib treatment restored their blood pDCs both quantitatively and functionally compared with healthy donors (40). While imatinib treatment did not affect $\mathrm{pDC}$ numbers in the peripheral circulation of patients with SSc, we demonstrate a significant reduction in $\mathrm{pDCs}$ in the BAL 1 year after imatinib treatment. Thus, imatinib may modulate the trafficking of $\mathrm{pDCs}$ and restore their homeostasis in disease states. In resonance with this hypothesis, $\triangle \mathrm{pDC}$ correlated with changes in local levels of proteins involved in cellular recruitment/chemotaxis (Supplemental Figure 10). After imatinib treatment, the $\triangle \mathrm{pDC}$ also correlated with changes in BAL levels of PAI1-a, as well as with FGFb, IL-17, and IL-1R1, all of which can promote extracellular matrix deposition (Supplemental Figure 10). Taken together, these observations raise the possibility of a novel mechanism: that imatinib might ameliorate fibrosis by restoring the homeostasis of pDCs.

We are cognizant of the limitations of this study. First, although the anti-PDCA-1 Ab treatment did not reduce other DC subsets, T, B, and NK cells in the spleen of naive and

bleomycin-injected B6 mice (Figure 4, Supplemental Figures 1 and 2), one cannot exclude some unknown, off-target effects of anti-PDCA-1 Ab. Complementary gene-targeting approaches would be helpful to further address the role of pDCs in fibrosis. Second, the bleomycin model, like other animal models, does not replicate all aspects of SSc (41). A rapid acceleration of lung disease in this model diminishes its utility for testing interventions to treat chronic fibrotic diseases. Third, although $\mathrm{pDC}$ depletion led to differential expression of profibrotic and proinflammatory cytokines, it is unclear whether the cytokines directly elaborated from pDCs play a role in fibrosis. Fourth, results of human studies need to be validated using a larger sample size. Finally, our results do not explicitly incriminate pDCs as the primary driver of fibrogenesis. Since pDCs produce proinflammatory cytokines (28), they may play a role in fibrosis via promoting inflammation; depletion of pDCs will attenuate such inflammation, thus limiting a cascading event that culminates in fibrosis. Under certain conditions, pDCs can also suppress aberrant immune response and inflammation through induction of immune suppressive cytokines and Tregs $(42,43)$. Hence, it is possible to speculate that $\mathrm{pDCs}$ traffic to the affected organs to quell inflammation via producing or inducing the production of immunosuppressive cytokines such as TGF $\beta 1$ and IL-4, which are also known to promote local tissue repair; this repair, when uncontrolled, leads to fibrosis. Consistent with this idea, pDC frequencies correlated with type 2 cytokines and TGF $\beta 1$. Nonetheless, our findings implicate pDCs in the pathogenesis of systemic fibrosis.

In conclusion, we present several lines of direct and correlative evidences in an animal model and in humans with the disease, which implicate pDCs in the pathogenesis of systemic fibrosis. The frequency of $\mathrm{pDC}$ at the disease site correlated with disease parameters in patients with SSc, and $\mathrm{pDC}$ depletion prevented bleomycin-induced fibrosis in animals. Our observations also suggest potential mechanisms by which pDCs 
Blood

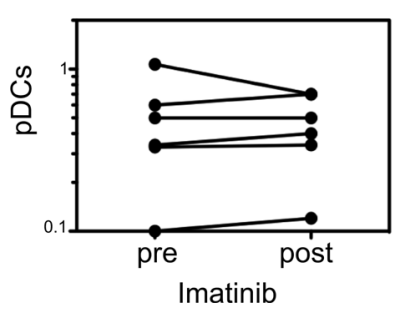

BAL

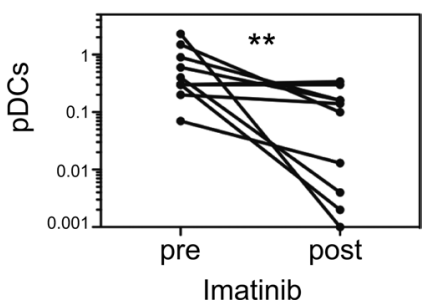

Figure 10. Effect of treatment with imatinib on PDCs in peripheral blood and BAL in patients with SSc. Frequencies of pDCs in blood and BAL were compared at baseline and 1 year after imatinib. Each line represents values from a single patient. Pre, baseline; Post, after imatinib. ${ }^{* *} P=0.004$, Wilcoxon signed-rank test.

might promote fibrosis. While the exact mechanism underlying pDC's effect in SSc remains to be elucidated, our results offer $\mathrm{pDC}$ manipulation as a potential therapeutic target in SSc and other fibrotic diseases.

\section{Methods}

Animals. C57BL/6 mice, all 8- to 10-week-old females, were purchased from the Jackson Laboratory.

Human subjects. Human samples from 71 subjects were used in this study (Supplemental Table 1). Peripheral blood samples were obtained from 15 SSc patients and 15 healthy donors. Major exclusion criteria included active infection, cancer, and recent (within 3 months) use of prednisone (>10 mg daily) and other immune suppressive agents.

BAL samples were obtained from 15 SSc patients who were participants in a clinical trial of imatinib for SSc-ILD (18). Patient characteristics included disease duration $\leq 7$ years, FVC $<85 \%$ predicted and $\geq 50 \%$ predicted, dyspnea on exertion, and presence of GGO on HRCT. Major exclusion criteria included FVC $<50 \%$, FEV1/FVC ratio $<65 \%$, abnormalities on HRCT not attributable to SSc-ILD, and pulmonary hypertension requiring treatment. BAL samples from 6 patients who had bronchoscopy for respiratory symptoms but were found to have no significant lung disease on further evaluations, including imaging and/or biopsy, were used as controls.

Punch skin biopsies were collected from 14 subjects, including 8 patients with early SSc ( $<5$ years since first non-Raynaud symptoms) and 6 heathy volunteers. Lung tissue sections were obtained from 15 subjects, including 8 SSc lung explants with pulmonary fibrosis and pulmonary hypertension and 7 control lungs.

Induction and assessment of bleomycin-induced fibrosis. Animals were injected with bleomycin (MilliporeSigma) $100 \mu \mathrm{g}$ (4 U per kg body weight) s.c. daily for 2 consecutive weeks. Animals were monitored daily for any clinical features including alopecia, submandibular erythema, hunchback appearance, and piloerection. A clinical disease severity score was assessed, where each of the aforementioned features were given a score of 1 , as described previously $(44,45)$.

For histopathological evaluation of fibrosis, lungs and submandibular skin were harvested on day 28 and fixed in 10\% neutral phosphate-buffered formalin. The paraffin-embedded tissues were cut into $5-\mu \mathrm{m}$ sections and stained with H\&E or Masson's trichrome. Fibrosis was assessed using a modified Ashcroft score (46), a numerical fibrosis scoring scale, in Masson's trichrome-stained sections. A score of 0 was considered as no fibrosis, 1 as minimal, 2 as mild, 3 as moderate, and 4 as severe fibrosis. Grading was performed by 2 investigators in a blinded fashion. Dermal thickness was assessed at 3 different locations on the same slide. An average value was used to represent findings pertaining to each tissue.

In vivo depletion of $p D C$ s. Animals were injected with anti-PDCA-1 Ab (clone JF05-IC2.41; Miltenyi Biotec) i.p. $100 \mathrm{~g}$ each on days -1 and 14 . Control animals received the same regimen of an isotype-matched $\mathrm{Ab}$ (IgG2b; clone ES26-5E12.4; Miltenyi Biotec). The efficiency of depletion was assessed in the lymphoid organs and lung by flow cytometry for pDCs (CD11 $\mathrm{c}^{\mathrm{int} / \mathrm{low}} \mathrm{B} 220^{+} \mathrm{PDCA}-1^{+}$). In some experiments, anti-PDCA-1 Ab was injected on days 7 and 14 (200 $\mu \mathrm{g}$ each) or on days 7, 10, and $14(100 \mu \mathrm{g}$ each).

Measurement of collagen content in the skin and lung tissue. Lung and submandibular skin tissues harvested from animals were homogenized and the soluble collagen content determined in tissue homogenates using the Sircol Soluble Collagen Assay Kit (Biocolor Ltd.) according to the manufacturer's instructions. Total protein was assessed using the Pierce BCA Protein Assay Kit in 96-well-plate format. 
Picrosirius red stain to assess total and fibrillary collagen. Paraffin sections $(5 \mu \mathrm{m})$ were stained with $0.1 \%$ Picrosirius red (Direct Red 80, MilliporeSigma) to reveal fibrillar collagen. The sections were serially imaged using parallel and orthogonal light. Microscope conditions (lamp brightness, condenser opening, objective, zoom, exposure time, and gain parameters) were maintained throughout the imaging of all samples.

RNA extraction and semiquantitative reverse transcription (RT) PCR. Lung and skin tissues were harvested and immediately submerged in RNAlater Stabilization Solution (Invitrogen). Total RNA was isolated using Trizol reagent (Invitrogen). RNA obtained from each sample (1 $\mu \mathrm{g})$ was reverse transcribed to cDNA using the RT ${ }^{2}$ First Strand Kit (Qiagen). The PCR array analysis of 83 genes included in the Mouse Dendritic and Antigen Presenting Cell RT² Profiler PCR Array (PAMM-406ZA, Qiagen) using a iQ5 system (Bio-Rad), according to the manufacturer's instructions. Heatmaps of all genes covered in this array are shown in Supplemental Figures 11 and 12. The raw array data were processed and analyzed using the $\triangle \mathrm{Ct}$ at the PCR Array Data Analysis System (http://saweb2.sabiosciences. $\mathrm{com} / \mathrm{pcr} /$ arrayanalysis.php). Fold changes were calculated and expressed as the log-normalized ratios of the experimental/control groups.

ELISA. Lung and skin tissues were lysed in a medium containing $20 \mathrm{mM}$ Tris- $\mathrm{HCl}$ (pH 7.5), $150 \mathrm{mM}$ $\mathrm{NaCl}, 5 \mathrm{mM}$ EDTA, $0.5 \%$ of Triton X-100, $1 \mathrm{mM}$ Na3VO4, Protease Inhibitor Cocktail (P8340, MilliporeSigma), and $1 \mathrm{mM}$ PMSF and centrifuged at 21,000 $\mathrm{g}$ for 20 minutes. The lysate supernatants were assayed for total protein content using the Pierce BCA protein assay kit. Tissue lysates were analyzed for TGF $\beta 1$, CXCL12, and CCL2, according to the manufacturer's instructions (Supplemental Methods).

Western blot. Protein lysate were prepared as described above. Protein for each sample $(50 \mu \mathrm{g})$ was loaded to individual lanes. Proteins were separated by electrophoresis in SDS-PAGE and transferred to PVDF membranes. The membranes were used for immunedetection of TLR7, C/EBP $\alpha$, STAT3, Rac-1 TSP-1, LRP1, and $\beta$-actin (Supplemental Methods). Blots were imaged using Bio-Rad Quantity One gel imaging software and quantitated using NIH Image J software.

Imatinib treatment. Imatinib was initiated at a dose of $100 \mathrm{mg}$ per day (18) and increased by $100 \mathrm{mg}$ every 2 weeks to a maximum dose of $600 \mathrm{mg}$ per day. Patients were allowed low-dose prednisone $(<10 \mathrm{mg}$ per day) or equivalent during the trial. Patients were required to discontinue any putative disease-modifying treatment for SSc at least 4 weeks before the study.

Assessment of lung fibrosis in patients with SSc. HRCT scans were scored by a radiologist blinded to clinical information, as described in Supplemental Figure 7. A linear scoring system was employed that accounted for the percentage of area affected in each lobe separately for the extent of parenchymal abnormalities including GGO and fibrosis. This scoring system is based on that reported $(47,48)$ but differs by scoring to the closest percentage and not the quartiles.

Analysis of BAL cells. BAL was collected from RML and RLL before and after imatinib treatment. BAL cells were stained for cell surface markers of DC and T cells followed by intracellular staining for cytokines.

Statistics. Differences in scores between the 2 groups were determined by nonparametric Mann-Whitney $U$ test. For multiple comparisons, we used 1-way ANOVA with Newman-Keuls test. For comparing categorical data, analysis was performed by creating contingency tables and applying $\chi^{2}$ test. Two-tailed $t$ tests were performed to compare parametric data. Spearman nonparametric correlation analysis was performed to determine correlations between cell frequencies and disease parameters. Correlation values were interpreted as follows: $0.00-0.20$, no correlation; $0.21-0.40$, low; 0.41-0.60, moderate; $0.61-0.80$, marked; and 0.81-1.00, high correlations (49). Wilcoxon signed-rank test was used to compare baseline and postimatinib values. For gene array data multiple comparisons, we used a FDR approach using the 2-stage linear step-up procedure of Benjamini, Krieger, and Yekutieli, with a desired FDR $(q)$ of $10 \%(50)$.

Gene function network analysis of differentially expressed genes was performed using GeneMANIA, a biological network integration algorithm that shows the relationships between genes weighting the molecular function based on their protein domain attributes (51) (Supplemental Figure 5).

Study approval. All human and animal studies were performed in accordance with the approved institutional guidelines and protocol from the University of California at Los Angeles. Written informed consent was received from human subjects prior to inclusion in the study.

Additional information is provided in Supplemental Methods. 


\section{Author contributions}

SK, IV, and AAD performed all experiments, analyzed data, and assisted with writing the manuscript; RS performed bronchocsopies and collected BAL samples; FA quantitated HRCT scans; DEF and DK performed the clinical trial and contributed to data interpretation and manuscript editing; RRS contributed to study design, data analysis and interpretation, wrote the manuscript, and supervised all aspects of this study.

\section{Acknowledgments}

This work was supported in part by grants from the NIH, R01AI80778 (RRS), R01AR055075 (RRS), R01AR56465 (RRS), K01 HL118712 (IV), and K23 AR053858 and K24 AR063120 (DK). SK and IV were supported by NIH T32 AR053463 (RRS). AAD and SK were supported by Reentry Supplement grants NIH U01AR55057-01S1 (DEF) and 4R01AI080778-04S1 (RRS), respectively. We thank Dora Acuna, Pallavi Gangalum, and Ivan Lopez for technical assistance; Philip Clements for helpful suggestions; and David Elashoff, Daniela Markovic, and Tristan Grogan at the UCLA CTSI Biostatistics Program (NIH-NCATS UL1 TR000124) for assisting with statistical analyses.

Address correspondence to: Ram Raj Singh, UCLA Division of Rheumatology, 1000 Veteran Ave, 32-59, Los Angeles, California 90095-1670, USA. Phone: 310.206.7491; Email: RRSingh@mednet.ucla.edu.

AAD's present address is: Department of Cellular Analysis, BioLegend, San Diego, California, USA.

DK's present address is: University of Michigan Scleroderma Program, Ann Arbor, Michigan, USA.

1. Saxena V, et al. Dual roles of immunoregulatory cytokine TGF-beta in the pathogenesis of autoimmunity-mediated organ damage. J Immunol. 2008;180(3):1903-1912.

2. Singh RR. SLE: translating lessons from model systems to human disease. Trends Immunol. 2005;26(11):572-579.

3. Wynn TA. Fibrotic disease and the T(H)1/T(H)2 paradigm. Nat Rev Immunol. 2004;4(8):583-594.

4. Divekar AA, et al. Treatment with imatinib results in reduced IL-4-producing T cells, but increased CD4(+) T cells in the broncho-alveolar lavage of patients with systemic sclerosis. Clin Immunol. 2011;141(3):293-303.

5. Greenblatt MB, Aliprantis AO. The immune pathogenesis of scleroderma: context is everything. Curr Rheumatol Rep. 2013;15(1):297.

6. Marchal-Sommé J, et al. Dendritic cells accumulate in human fibrotic interstitial lung disease. Am J Respir Crit Care Med. 2007;176(10):1007-1014.

7. Xie Y, Zhang X, Inoue Y, Wakasugi S, Makino T, Ihn H. Expression of CD1a and CD86 on scleroderma Langerhans cells. Eur J Dermatol. 2008;18(1):50-54.

8. van Bon L, et al. Distinct evolution of TLR-mediated dendritic cell cytokine secretion in patients with limited and diffuse cutaneous systemic sclerosis. Ann Rheum Dis. 2010;69(8):1539-1547.

9. Bantsimba-Malanda C, et al. A role for dendritic cells in bleomycin-induced pulmonary fibrosis in mice? Am J Respir Crit Care Med. 2010;182(3):385-395.

10. Chia JJ, et al. Dendritic cells maintain dermal adipose-derived stromal cells in skin fibrosis. J Clin Invest. 2016;126(11):4331-4345.

11. King JK, et al. Langerhans Cells Maintain Local Tissue Tolerance in a Model of Systemic Autoimmune Disease. J Immunol. 2015;195(2):464-476.

12. van Bon L, et al. Proteome-wide analysis and CXCL4 as a biomarker in systemic sclerosis. N Engl J Med. 2014;370(5):433-443.

13. Divekar AA, et al. pDCs and IL-4+ T-cells in scleroderma as novel targets of imatinib mesylate (44.18). J Immunol. 2011;186(1 Supplement):44.18.

14. Wolf D, Rumpold H. A benefit-risk assessment of imatinib in chronic myeloid leukaemia and gastrointestinal stromal tumours. Drug Saf. 2009;32(11):1001-1015.

15. Dewar AL, Zannettino AC, Hughes TP, Lyons AB. Inhibition of c-fms by imatinib: expanding the spectrum of treatment. Cell Cycle. 2005;4(7):851-853.

16. Soria A, et al. The effect of imatinib (Glivec) on scleroderma and normal dermal fibroblasts: a preclinical study. Dermatology (Basel). 2008;216(2):109-117.

17. Daniels CE, et al. Imatinib mesylate inhibits the profibrogenic activity of TGF-beta and prevents bleomycin-mediated lung fibrosis. J Clin Invest. 2004;114(9):1308-1316.

18. Khanna D, et al. A one-year, phase I/IIa, open-label pilot trial of imatinib mesylate in the treatment of systemic sclerosis-associated active interstitial lung disease. Arthritis Rheum. 2011;63(11):3540-3546.

19. Spiera RF, et al. Imatinib mesylate (Gleevec) in the treatment of diffuse cutaneous systemic sclerosis: results of a 1-year, phase IIa, single-arm, open-label clinical trial. Ann Rheum Dis. 2011;70(6):1003-1009.

20. Fraticelli P, et al. Low-dose oral imatinib in the treatment of systemic sclerosis interstitial lung disease unresponsive to cyclophosphamide: a phase II pilot study. Arthritis Res Ther. 2014;16(4):R144.

21. Kumar R, et al. TGF- $\beta$ activation by bone marrow-derived thrombospondin-1 causes Schistosoma- and hypoxia-induced pulmonary hypertension. Nat Commun. 2017;8:15494

22. Hansell DM. High-resolution computed tomography in the evaluation of fibrosing alveolitis. Clin Chest Med. 1999;20(4):739-60. 
23. Remy-Jardin M, Giraud F, Remy J, Copin MC, Gosselin B, Duhamel A. Importance of ground-glass attenuation in chronic diffuse infiltrative lung disease: pathologic-CT correlation. Radiology. 1993;189(3):693-698.

24. Tashkin DP, et al. Effects of 1-year treatment with cyclophosphamide on outcomes at 2 years in scleroderma lung disease. Am $J$ Respir Crit Care Med. 2007;176(10):1026-1034.

25. Goh NS, et al. Interstitial lung disease in systemic sclerosis: a simple staging system. Am J Respir Crit Care Med. 2008;177(11):1248-1254.

26. Gordon J, et al. Imatinib mesylate (Gleevec) in the treatment of diffuse cutaneous systemic sclerosis: results of a 24-month open label, extension phase, single-centre trial. Clin Exp Rheumatol. 2014;32(6 Suppl 86):S-189.

27. Fancke B, Suter M, Hochrein H, O'Keeffe M. M-CSF: a novel plasmacytoid and conventional dendritic cell poietin. Blood. 2008;111(1):150-159.

28. Swiecki M, Colonna M. The multifaceted biology of plasmacytoid dendritic cells. Nat Rev Immunol. 2015;15(8):471-485.

29. Umemoto E, et al. Constitutive plasmacytoid dendritic cell migration to the splenic white pulp is cooperatively regulated by CCR7- and CXCR4-mediated signaling. J Immunol. 2012;189(1):191-199.

30. Welner RS, et al. C/EBP $\alpha$ is required for development of dendritic cell progenitors. Blood. 2013;121(20):4073-4081.

31. Zhong G, et al. Enhanced maturation and functional capacity of dendritic cells induced by mannosylated L2 domain of ErbB2 receptor. Scand J Immunol. 2005;62(2):108-116.

32. Hashizume H, Horibe T, Yagi H, Seo N, Takigawa M. Compartmental imbalance and aberrant immune function of blood CD123+ (plasmacytoid) and CD11c+ (myeloid) dendritic cells in atopic dermatitis. J Immunol. 2005; 174(4):2396-2403

33. Ghirelli C, Zollinger R, Soumelis V. Systematic cytokine receptor profiling reveals GM-CSF as a novel TLR-independent activator of human plasmacytoid predendritic cells. Blood. 2010;115(24):5037-5040.

34. Walsh ER, et al. Strain-specific requirement for eosinophils in the recruitment of $\mathrm{T}$ cells to the lung during the development of allergic asthma. J Exp Med. 2008;205(6):1285-1292.

35. Mashikian MV, et al. Identification of IL-16 as the lymphocyte chemotactic activity in the bronchoalveolar lavage fluid of histamine-challenged asthmatic patients. J Allergy Clin Immunol. 1998;101(6 Pt 1):786-792.

36. Huaux F, Liu T, McGarry B, Ullenbruch M, Phan SH. Dual roles of IL-4 in lung injury and fibrosis. J Immunol. 2003;170(4):2083-2092.

37. Singh RR. IL-4 and many roads to lupuslike autoimmunity. Clin Immunol. 2003;108(2):73-79.

38. Ebner S, et al. A novel role for IL-3: human monocytes cultured in the presence of IL-3 and IL- 4 differentiate into dendritic cells that produce less IL-12 and shift Th cell responses toward a Th2 cytokine pattern. J Immunol. 2002;168(12):6199-6207.

39. Chakraborty D, et al. Activation of STAT3 integrates common profibrotic pathways to promote fibroblast activation and tissue fibrosis. Nat Commun. 2017;8(1):1130.

40. Mohty M, et al. Imatinib and plasmacytoid dendritic cell function in patients with chronic myeloid leukemia. Blood. 2004;103(12):4666-4668.

41. Marangoni RG, Varga J, Tourtellotte WG. Animal models of scleroderma: recent progress. Curr Opin Rheumatol. 2016;28(6):561-570.

42. Kavousanaki M, Makrigiannakis A, Boumpas D, Verginis P. Novel role of plasmacytoid dendritic cells in humans: induction of interleukin-10-producing Treg cells by plasmacytoid dendritic cells in patients with rheumatoid arthritis responding to therapy. Arthritis Rheum. 2010;62(1):53-63.

43. de Heer HJ, et al. Essential role of lung plasmacytoid dendritic cells in preventing asthmatic reactions to harmless inhaled antigen. J Exp Med. 2004;200(1):89-98.

44. Burkholder T, Foltz C, Karlsson E, Linton CG, Smith JM. Health Evaluation of Experimental Laboratory Mice. Curr Protoc Mouse Biol. 2012;2:145-165.

45. Yang D, et al. Inducible Costimulator Gene-Transduced Bone Marrow-Derived Mesenchymal Stem Cells Attenuate the Severity of Acute Graft-Versus-Host Disease in Mouse Models. Cell Transplant. 2015;24(9):1717-1731.

46. Hübner RH, et al. Standardized quantification of pulmonary fibrosis in histological samples. BioTechniques. 2008;44(4):507-511.

47. Gay SE, et al. Idiopathic pulmonary fibrosis: predicting response to therapy and survival. Am J Respir Crit Care Med. 1998;157(4 Pt 1):1063-1072.

48. Zisman DA, et al. High-resolution chest CT findings do not predict the presence of pulmonary hypertension in advanced idiopathic pulmonary fibrosis. Chest. 2007;132(3):773-779.

49. Franzblau AN. A primer of statistics for non-statisticians. New York, NY: Harcourt Brace; 1958.

50. Benjamini Y, Krieger AM, Yekutieli D. Adaptive linear step-up procedures that control the false discovery rate. Biometrika. 2006;93(3):491-507.

51. Mostafavi S, Ray D, Warde-Farley D, Grouios C, Morris Q. GeneMANIA: a real-time multiple association network integration algorithm for predicting gene function. Genome Biol. 2008;9 Suppl 1:S4. 